\title{
On the theory of Gordan-Noether on homogeneous forms with zero Hessian (improved version)
}

\author{
Junzo Watanabe \\ Department of Mathematics \\ Tokai University \\ Hiratsuka, Kanagawa 259-1292, Japan \\ Email:watanabe.junzo@tokai-u.jp \\ Michiel de Bondt \\ Institute for Mathematics, Astrophysics and Particle Physics \\ Radboud University Nijmegen, The Netherlands \\ Email:M.deBondt@math.ru.nl
}

\begin{abstract}
An earlier version of this paper, namely [18], has been printed in Proceedings of the School of Science of Tokai University, Vol. 49, Mar. 2014. In this version, a serious error has been corrected, namely [18, Lemma 5.2]. Lemma 5.2 has been replaced by a weaker statement, and Proposition 6.2 has been weakened along with that. Lemma 5.2 no longer suffices for the proof of Proposition 7.2. For that reason, a new section (Section 8) has been added to complete the proof of Proposition 7.2. Furthermore, several trivial errors have been corrected, and a section with new results has been added (Section 9).

Abstract

We give a detailed proof for Gordan-Noether's results in "Ueber die algebraischen Formen, deren Hesse'sche Determinante identisch verschwindet." C. Lossen has written a paper in a similar direction as the present paper, but did not provide a proof for every result. In our paper, every result is proved. Furthermore, our paper is independent of Lossen's paper and includes a considerable number of new observations.
\end{abstract}

\section{Contents}

1 Introduction $\quad 2$

2 Notation and preliminaries 5

3 Self-vanishing systems of polynomials

4 Forms with zero Hessian and reduced self-vanishing systems 13

5 Binary and ternary forms with zero Hessian 15

6 The rational map defined by $h \quad 17$ 
$7 \quad$ Quaternary and quinary forms with zero Hessian

8 Proof of $\operatorname{dim} W \leq 1$ in the proof of Proposition 7.2

25

9 Section 6 of Gordan and Noether [11]

31

\section{Introduction}

In 1852 and 1859, O. Hesse wrote two papers in Crelle's Journal Bd. 42 and Bd. 56 in which he claimed that if the Hessian determinant of a homogeneous polynomial identically vanishes, then a variable can be eliminated by a linear transformation of the variables. Unfortunately his claim is not true in general. In fact Hesse's proof was unconventional and the validity of the proof was questioned from the beginning [11]. Nonetheless it should have been easy to see that Hesse's claim is true for binary forms as well as quadrics. In 1875, M. Pasch proved that Hesse's claim is true for ternary cubics and quaternary cubics [15].

In 1876, P. Gordan and M. Noether [11] finally established the correct statement which says that if a form has zero Hessian, then one variable can be eliminated from the form itself and its partial derivatives simultaneously by way of a birational transformation of the variables. Moreover they proved in the same paper that Hesse's claim is true, if the number of variables is at most four, and furthermore they determined all homogeneous polynomials in five variables for which the Hessian determinant identically vanishes.

The present paper goes beyond the necessity and desire to understand their proof. The Hessian of a homogeneous polynomial is essential to the theory of Artinian Gorenstein rings because it is used with higher Hessians to determine the set of the strong Lefschetz elements in a zero-dimensional Gorenstein algebra ([14]). In particular, if the Hessian of a homogeneous polynomial is identically zero, we get a Gorenstein algebra which lacks the strong Lefschetz property. To explain this further, let $R=K\left[x_{1}, \ldots, x_{n}\right]$ be the polynomial ring over a field $K$ of characteristic zero and let $G \in R$ be a homogeneous polynomial. In addition let $I \subset R$ be the ideal:

$$
I=\left\{f\left(x_{1}, \ldots, x_{n}\right) \in R \mid f\left(\left(\frac{\partial}{\partial x_{1}}, \ldots, \frac{\partial}{\partial x_{n}}\right) G\right)=0\right\},
$$

and let $A=R / I$. Then $A$ is a zero-dimensional Gorenstein graded algebra $A=\bigoplus_{i=0}^{d} A_{i}$, where $d=\operatorname{deg} G$. It is easy to see that if the polynomial $G$ contains properly $n$ variables, then the partial derivatives $G_{1}, \ldots, G_{n}$ of $G$ are linearly independent. Moreover if $L=$ $\xi_{1} x_{1}+\cdots+\xi_{n} x_{n} \in R_{1}$ is a linear form, it defines a linear map

$$
\times L^{d-2}: A_{1} \longrightarrow A_{d-1}
$$

by $A_{1} \ni a \mapsto a L^{d-2} \in A_{d-1}$. Let $M$ be the matrix for this linear map with respect to the bases $\left\langle x_{1}, \ldots, x_{n}\right\rangle$ and $\left\langle G_{1}, \ldots, G_{n}\right\rangle$ for $A_{1}$ and $A_{d-1}$ respectively. Then $\operatorname{det} M$ is the Hessian of $G$ evaluated at $\left(\xi_{1}, \ldots, \xi_{n}\right)$ (up to a constant multiple). If $\operatorname{dim} A_{1} \leq 4$, and 
if we take the results of [11] for granted, this is to say that there exists a linear form $L$ such that $L^{d-2}$ is bijective. If $n=5$, Gordan-Noether's paper enables us to determine all homogeneous polynomials $G$ such that $L^{d-2}$ is not bijective for any choice of $\xi_{1}, \ldots, \xi_{n}$.

Gordan-Noether's paper [11] has been cited by several authors ([2], 12], 14], [17]); however each time it had to be accompanied with a proviso that the result is yet to be confirmed. Gordan-Noether's paper is difficult to understand. Not only their results but also the methods have been completely forgotten. Thus, it seems necessary to consider the paper from the viewpoint of contemporary algebra. H. Yamada [19] devoted considerable efforts to constructing a modernized translation of [11]; however, it was not completely successful and therefore it was unpublished.

The purpose of this paper is to give detailed proofs for most of the results that were obtained by Gordan-Noether in [11]. The foundation of their theory lies in the fact that if the Hessian determinant of a homogeneous polynomial is identically zero, then the polynomial satisfies a certain linear partial differential equation. For simplicity we assume that the Hessian matrix $M:=\left(\partial^{2} f / \partial x_{i} \partial x_{j}\right)$ has corank one. Then the left null space of the matrix $M$ has dimension one over the function field $K(x)$. Let $\left(h_{1}, \ldots, h_{n}\right)$ be a vector with $h_{j} \in K(x)$ such that $\left(h_{1}, \ldots, h_{n}\right) M=0$. Then it is easy to see that $f$ and its partial derivatives satisfy the partial differential equation

$$
h_{1} \frac{\partial F}{\partial x_{1}}+h_{2} \frac{\partial F}{\partial x_{2}}+\cdots+h_{n} \frac{\partial F}{\partial x_{n}}=0 .
$$

By clearing the denominator we may think $h_{j}$ are homogeneous polynomials of the same degree. (Cf. Remark 4.6.) Gordan and Noether discovered that each coefficient $h_{j}$ itself of (1) satisfies the partial differential equation (11). This readily proves that a variable can be eliminated from $f$ and its partial derivatives simultaneously by a birational transformation. (See Theorem 4.7.) The Gordan-Noether called the functions satisfying (11) "die Functionen $\Phi . "$ The coefficients of this partial linear differential equation were termed as a "self-vanishing system" by Yamada [19]. The solution of this type of differential equation behaves as if the coefficients were constants. According to [11], Jacobi considered this type of differential equation and it is the key to understanding the Gordan and Noether theory.

To prove that Hesse's claim is true for homogeneous polynomials for $n \leq 4$, it is necessary to consider the fundamental locus and the image of the rational map $\mathbb{P}^{n-1} \rightarrow$ $\mathbb{P}^{n-1}$ defined by $\left(x_{1}, \ldots, x_{n}\right) \mapsto\left(h_{1}, \ldots, h_{n}\right)$. From the fact that $h_{1}, \ldots, h_{n}$ is a "selfvanishing system," it follows that the dimension of the image is at most $n-3$ if $n \geq 3$. This enables us to determine the forms with zero Hessian for $n \leq 4$. For $n=5$, the dimension of the image of $h_{1}, \ldots, h_{n}$ may be exactly $n-3=2$, which is too large to determine the forms with zero Hessian in dimension 5. But this cannot occur in the context of forms with zero Hessian. So the dimension is at most 1 and we can determine the forms with zero Hessian for $n=5$ as well.

Gordan and Noether's idea and proof techniques appear quite new and they are interesting in their own right, and they give us a series of new problems, some of which will be investigated in our subsequent papers. 


\section{Historical notes}

In 1990, H. Yamada wrote a paper [19], under Grant-in-Aid no. 20022551 entitled "On the hypersurface defined by a form whose Hessian identically vanishes." It was unpublished because it was incomplete; however, he defined and systematically studied "the self-vanishing system of polynomials," as he named them. This paper was written to finish Yamada's paper [19].

An earlier version of this paper was written by the first author only without a prior knowledge of Lossen's paper [13. The first author gave a 3 hour lecture on this subject at the workshop "Aspects of SLP and WLP" held in Hawaii Tokai International College in Honolulu in September 2012, where he learned that Lossen had written a paper [13] in the same direction and that there were other related papers [6] and [8]. Clearly the objectives of Lossen's paper and this paper are identical. However the methods employed are different, although both are based on the same source, i.e., on the paper of Gordan and Noether [11].

The main differences with Lossen's paper are listed below.

1. Theorem 4.7 is not explicitly written in Lossen [13], which tells us that Hesse was in some sense correct in his intuition, when he said that if the Hessian determinant vanishes, then a variable can be eliminated by a linear transformation.

2. In this paper, a so-called "self-vanishing system" is defined and studied systematically.

3. Our observation Proposition 6.2 considerably simplify the entire argument.

4. In Lossen's paper [13], the connection to the Lefschetz properties of Artinian rings is not indicated.

5. In this paper it is proved that a cubic form in five variables is essentially unique, while in [13], the provided proof is incomplete.

In 2014, the first author published a version of this paper in Proceedings of the School of Science of Tokai University. But Lemma 5.2 of that paper contains a serious error. The error can however be fixed, and the second author has been added to the paper to do this. In the current version, Lemma 5.2 has been replaced by a weaker statement. Proposition 6.2 has been replaced along with that, and section 8 has been added to complete the proof of Proposition 7.2 (the new version of Lemma 5.2 does not suffice for this). Section 9 is due to the second author as well, and was inspired by the final remark (Remark 7.6) in the 2014 version of the paper.

The second author has written some related papers on his own. In [3], the forms with zero Hessian are determined for $n \leq 4$. In [4], using results of [2], the forms with zero Hessian are determined for $n=5$. But the proofs diverge from the techniques in Gordan-Noether's paper 11] on some points (see also remark 8.8). Non-homogeneous polynomials with zero Hessians are considered in [2] and [3] as well. In [5], all these 
results are generalized to arbitrary dimension, with the zero Hessian condition replaced by that the Hessian matrix has fixed small rank (one less than the original dimension).

The first author would like to thank H. Nasu and T. Tsukioka for insightful discussions for Remark 7.5.

\section{Notation and preliminaries}

Throughout the paper we denote by $K$ an algebraically closed field of characteristic zero. We denote by $K\left[x_{1}, x_{2}, \ldots, x_{n}\right]$ the polynomial ring in the variables $x_{1}, \ldots, x_{n}$ and by $K\left(x_{1}, \ldots, x_{n}\right)$ the function field. Let $R=K\left[x_{1}, \ldots, x_{n}\right]$. An element of $R$ is sometimes abbreviated as $f(x)$ or simply as $f$.

A system of homogeneous polynomials (or forms) of $R$ is a vector $\left(f_{1}, f_{2}, \ldots, f_{n}\right)$ consisting of homogeneous polynomials $f_{i} \in R$ of the same degree. A system of forms is denoted by a bold face letter as $\boldsymbol{f}=\left(f_{1}, \ldots, f_{n}\right)$. To avoid triviality we will always assume that $\boldsymbol{f} \neq 0$. To indicate that a system is a vector of polynomials depending on the argument vector $x=\left(x_{1}, \ldots, x_{n}\right)$, we write $\boldsymbol{f}(x)$ as well as $\boldsymbol{f}$. Although a coordinate system like $x=\left(x_{1}, \ldots, x_{n}\right)$ is a "system of forms," we do not apply the rule to use a bold face letter to denote it. If $y=\left(y_{1}, y_{2}, \ldots, y_{n}\right)$ is a new set of variables and if $\boldsymbol{f}(x)$ is a system of forms in $R$, then $\boldsymbol{f}(y)$ obtained from $\boldsymbol{f}(x)$ by the substitution $x_{j} \mapsto y_{j}$ is a system of forms in $K\left[y_{1}, \ldots, y_{n}\right]$.

We treat vectors both as row vectors and as column vectors, so if $A=\left(a_{i j}\right)$ is an $n \times n$ matrix with $a_{i j} \in K$, and if $x=\left(x_{1}, \ldots, x_{n}\right)$ is a vector, then $y=A x$ means that $y=\left(y_{1}, \ldots, y_{n}\right)$ is a vector defined by

$$
y_{i}=\sum_{j=1}^{n} a_{i j} x_{j} .
$$

Likewise $y=x A$ means that

$$
y_{j}=\sum_{i=1}^{n} a_{i j} x_{i} .
$$

The same rule applies to systems of polynomials as well as coordinate systems. Thus, if $\boldsymbol{f}=\left(f_{1}, \ldots, f_{n}\right)$ is a system of forms, then $\boldsymbol{f}^{\prime}=A \boldsymbol{f}$ is a system of forms for any $n \times n$ invertible matrix $A$ over $K$.

Lemma 2.1. Let $K[x]=K\left[x_{1}, \ldots, x_{n}\right]$ be the polynomial ring and let $f(x)=f\left(x_{1}, \ldots, x_{n}\right) \in$ $K[x]$. Let $A=\left(a_{i j}\right) \in \mathrm{GL}(n, K)$, and put

$$
\left(x_{1}^{\prime}, x_{2}^{\prime}, \ldots, x_{n}^{\prime}\right)=A\left(x_{1}, x_{2}, \ldots, x_{n}\right)
$$

Consider $\left(x_{1}^{\prime} \ldots, x_{n}^{\prime}\right)$ as a new coordinate system and let $f^{\prime}$ be the polynomial in $x_{1}^{\prime}, \ldots, x_{n}^{\prime}$ defined by

$$
f^{\prime}\left(x^{\prime}\right):=f\left(A^{-1} x^{\prime}\right)=f(x) .
$$


Then

$$
\left(f_{1}^{\prime}, f_{2}^{\prime}, \ldots, f_{n}^{\prime}\right)={ }^{t} A^{-1}\left(f_{1}, f_{2}, \ldots, f_{n}\right),
$$

where $f_{j}=\frac{\partial f}{\partial x_{j}}$, and $f_{j}^{\prime}=\frac{\partial f^{\prime}}{\partial x_{j}^{\prime}}$.

Proof is left to the reader.

Lemma 2.2. Let $f(x)=f\left(x_{1}, \ldots, x_{n}\right)$ be a homogeneous polynomial of positive degree. Put $f_{i}=\frac{\partial f}{\partial x_{i}}$ and let $\boldsymbol{f}=\left(f_{1}, \ldots, f_{n}\right)$ be a system of polynomials. If $\operatorname{dim}_{K} \sum_{i=1}^{n} K f_{i}=s$, then $n-s$ variables can be eliminated from $f$ by means of a linear transformation of the variables. In other words, there exists an invertible matrix $A=\left(a_{i j}\right) \in \mathrm{GL}(n, K)$ such that, if we let $x^{\prime}=A x$, then the polynomial $f^{\prime}\left(x_{1}^{\prime}, \ldots, x_{n}^{\prime}\right)=f\left(A^{-1} x^{\prime}\right)$ does not depend on $x_{s+1}^{\prime}, x_{s+2}^{\prime}, \ldots, x_{n}^{\prime}$.

Proof. By assumption there exists an invertible matrix $B$ such that $B\left(f_{1}, f_{2}, \ldots, f_{n}\right)=$ $\left(f_{1}^{\prime}, f_{2}^{\prime}, \ldots, f_{s}^{\prime}, 0,0, \ldots, 0\right)$. Let $A$ be the matrix such that ${ }^{t} A^{-1}=B$, and put $x^{\prime}=A x$ and $f^{\prime}\left(x^{\prime}\right)=f\left(A^{-1} x^{\prime}\right)$. Then

$$
\frac{\partial f^{\prime}}{\partial x_{j}^{\prime}}=0 \text { for } j \geq s+1,
$$

by Lemma 2.1. Thus $f^{\prime}\left(x^{\prime}\right)$ does not depend on the variables $x_{s+1}^{\prime}, x_{s+2}^{\prime}, \ldots, x_{n}^{\prime}$.

Proposition 2.3. Let $\boldsymbol{f}=\left(f_{1}, \ldots, f_{n}\right)$ be a system of forms in $K\left[x_{1}, \ldots, x_{n}\right]$, where $f_{1}, \ldots, f_{n}$ are algebraically dependent. Let $y=\left(y_{1}, \ldots, y_{n}\right)$ be a new set of variables independent of $x$. Let

$$
\phi: K\left[y_{1}, y_{2}, \ldots, y_{n}\right] \rightarrow K\left[x_{1}, x_{2}, \ldots, x_{n}\right]
$$

be the homomorphism defined by $y_{i} \mapsto f_{i}$, and let $g=g\left(y_{1}, \ldots, y_{n}\right)$ be a nonzero element in ker $\phi$ of the least degree. Put

$$
h_{j}=h_{j}(x):=\frac{\partial g}{\partial y_{j}}\left(f_{1}, \ldots, f_{n}\right) .
$$

In words, $h_{j}(x)$ is the polynomial obtained from $\frac{\partial g}{\partial y_{j}}$ by substituting $\left(f_{1}, \ldots, f_{n}\right)$ for $\left(y_{1}, \ldots\right.$, $\left.y_{n}\right)$. Let $W=\sum_{j=1}^{n} K h_{j}$ be the vector space over $K$ spanned by the elements $h_{1}, \ldots, h_{n}$. Let $s=\operatorname{dim}_{K} W$. Then $n-s$ variables can be eliminated from $g(y)$ by means of a linear transformation of the variables $y_{1}, \ldots, y_{n}$.

Proof. Put $V=\sum_{i=1}^{n} K \frac{\partial g}{\partial y_{i}}$. We claim that $\operatorname{dim}_{K} V=s$. It is clear that $\operatorname{dim}_{K} V \geq s$. Consider the restriction

$$
\left.\phi\right|_{V}: V \rightarrow K\left[x_{1}, x_{2}, \ldots, x_{n}\right] .
$$

We see that $(\operatorname{ker} \phi) \cap V=0$ by minimality of the degree of $g$. Thus we have $\operatorname{dim}_{K} V=s$, since $\left.\phi\right|_{V}$ is injective and $\left.\operatorname{im} \phi\right|_{V}$ is $W$. By the previous lemma, proof is complete.

Remark 2.4. In the above Proposition, it is possible that $h_{j}=0$ for some $j$. It means that $\frac{\partial g}{\partial y_{j}}=0$. Hence $\boldsymbol{h}:=\left(h_{1}, \ldots, h_{n}\right) \neq 0$. If we drop the condition that deg $g$ is minimal in $\mathcal{I}$, we may define $\boldsymbol{h}$ as well, but $\boldsymbol{h}$ can be 0 . 


\section{Self-vanishing systems of polynomials}

Let $x=\left(x_{1}, \ldots, x_{n}\right), y=\left(y_{1}, \ldots, y_{n}\right)$ be two sets of indeterminates and let $K(x, y)=$ $K\left(x_{1}, \ldots, x_{n}, y_{1}, \ldots, y_{n}\right)$ denote the rational function field. We introduce the differential operator

$$
\mathcal{D}_{x}(y): K(x, y) \rightarrow K(x, y)
$$

which is defined by

$$
\mathcal{D}_{x}(y) f(x, y):=\sum_{j=1}^{n} y_{j} \frac{\partial f(x, y)}{\partial x_{j}}
$$

for $f(x, y) \in K(x, y)$. For a homogeneous polynomial $f(x)=f\left(x_{1}, \ldots, x_{n}\right) \in K\left[x_{1}, \ldots, x_{n}\right]$ and for $j \geq 0$, define $f^{(j)}(x, y)$ to be the polynomial in $K\left[y_{1}, \ldots, y_{n}, x_{1}, \ldots, x_{n}\right]$ given by

$$
f^{(j)}(x, y)=\frac{1}{j !} \mathcal{D}_{x}(y)^{j}(f(x)) .
$$

It is easy to see that

$$
f^{(j)}(x, x)=\left(\begin{array}{l}
d \\
j
\end{array}\right) f(x), \quad j=0,1,2, \cdots, d
$$

and

$$
f^{(d)}(x, y)=f(y)
$$

where $d=\operatorname{deg} f$.

Proposition 3.1. Let $A=\left(a_{i j}\right)$ be an invertible matrix with $a_{i j} \in K$ and let

$$
\begin{aligned}
x^{\prime} & =\left(x_{1}^{\prime}, \ldots, x_{n}^{\prime}\right)=A\left(x_{1}, \ldots, x_{n}\right), \\
y^{\prime} & =\left(y_{1}^{\prime}, \ldots, y_{n}^{\prime}\right)=A\left(y_{1}, \ldots, y_{n}\right) .
\end{aligned}
$$

Let $f^{\prime}\left(x^{\prime}\right)$ be the polynomial in the coordinate $x^{\prime}$ defined by $f^{\prime}\left(x^{\prime}\right)=f(x) \in K\left[x_{1}, \ldots, x_{n}\right]$. Then we have

$$
\mathcal{D}_{x^{\prime}}\left(y^{\prime}\right) f^{\prime}\left(x^{\prime}\right)=\mathcal{D}_{x}(y) f(x) .
$$

Proof. Let $\boldsymbol{f}$ be the system of forms:

$$
\boldsymbol{f}=\frac{\partial f(x)}{\partial x}:=\left(\frac{\partial f(x)}{\partial x_{1}}, \frac{\partial f(x)}{\partial x_{2}}, \ldots, \frac{\partial f(x)}{\partial x_{n}}\right) .
$$

Likewise, let $\boldsymbol{f}^{\prime}$ be the system of forms:

$$
\boldsymbol{f}^{\prime}=\frac{\partial f^{\prime}\left(x^{\prime}\right)}{\partial x^{\prime}}=\left(\frac{\partial f^{\prime}\left(x^{\prime}\right)}{\partial x_{1}^{\prime}}, \frac{\partial f^{\prime}\left(x^{\prime}\right)}{\partial x_{2}^{\prime}}, \ldots, \frac{\partial f^{\prime}\left(x^{\prime}\right)}{\partial x_{n}^{\prime}}\right) .
$$

Then, by definition,

$$
\mathcal{D}_{x}(y) f(x)=y \cdot \boldsymbol{f}=y_{1} \frac{\partial f}{\partial x_{1}}+\cdots+y_{n} \frac{\partial f}{\partial x_{n}} .
$$


By Lemma 2.1 we have

$$
\begin{aligned}
\mathcal{D}_{x}(y) f(x)=y \cdot \boldsymbol{f} & =\left(A^{-1} y^{\prime}\right) \cdot\left({ }^{t} A \boldsymbol{f}^{\prime}\right) \\
& =\left(y^{\prime}\left({ }^{t} A^{-1}\right)\right) \cdot\left({ }^{t} A \boldsymbol{f}^{\prime}\right) \\
& =y^{\prime} \cdot \boldsymbol{f}^{\prime}=\mathcal{D}_{x^{\prime}}\left(y^{\prime}\right) f^{\prime}\left(x^{\prime}\right) .
\end{aligned}
$$

Proposition 3.2. For $f(x) \in K[x]$, we have

$$
f(x+t y)=\sum_{j=0}^{\infty} t^{j} f^{(j)}(x, y)
$$

for an indeterminate $t$.

Proof. This is a direct consequence of the Taylor expansion.

Notation 3.3. Let $\boldsymbol{h}=\left(h_{1}(x), \ldots, h_{n}(x)\right)$ be a system of homogeneous polynomials $h_{i} \in K\left[x_{1}, \ldots, x_{n}\right]$. We define the differential operator $\mathcal{D}_{x}(\boldsymbol{h}): K(x) \rightarrow K(x)$ associated to $\boldsymbol{h}$ by

$$
\mathcal{D}_{x}(\boldsymbol{h}) f(x)=\sum_{j=1}^{n} h_{j}(x) \frac{\partial f(x)}{\partial x_{j}}=f^{(1)}(x, h(x)) .
$$

Furthermore we denote by

$$
\operatorname{Sol}(\boldsymbol{h} ; R)
$$

the set of solutions in $R \subseteq K[x]$ of the differential equation

$$
\mathcal{D}_{x}(\boldsymbol{h}) f(x)=0 \text {. }
$$

Namely,

$$
\operatorname{Sol}(\boldsymbol{h} ; R)=\left\{\begin{array}{l|l}
f(x) \in R & \sum_{j=1}^{n} h_{j}(x) \frac{\partial f(x)}{\partial x_{j}}=0
\end{array}\right\} .
$$

Note that $\operatorname{Sol}(\boldsymbol{h} ; K[x])$ is a graded subalgebra of $K[x]$.

Definition 3.4. A system $\boldsymbol{h}=\left(h_{1}(x), \ldots, h_{n}(x)\right)$ of polynomials is called self-vanishing, if $h_{j}(x) \in \operatorname{Sol}(\boldsymbol{h} ; K[x])$ for all $j=1,2, \ldots, n$. In addition to it, if $\operatorname{GCD}\left(h_{1}, \ldots, h_{n}\right)=1$, we will say that $\boldsymbol{h}$ is a reduced self-vanishing system.

Example 3.5. A constant vector $\boldsymbol{h}=\left(c_{1}, c_{2}, \ldots, c_{n}\right) \in K^{n}$ is obviously a self-vanishing system.

Example 3.6. Let $h_{j} \in K[x]$ be homogeneous polynomials (of the same degree). Suppose that $\boldsymbol{h}=\left(h_{1}, \ldots, h_{n}\right)$ satisfy the following conditions.

1. $h_{1}=\cdots=h_{r}=0$, for some integer $r ; 1 \leq r<n$. 
2. The polynomials $h_{r+1}, \ldots, h_{n}$ do not involve the variables $x_{r+1}, \ldots, x_{n}$.

Then $\boldsymbol{h}$ is a self-vanishing system of forms.

Definition 3.7. Let $\boldsymbol{f}=\left(f_{1}, \ldots, f_{n}\right)$ be a system of forms in $K[x]$. We denote by $\partial \boldsymbol{f} / \partial x_{j}$ the system of forms:

$$
\frac{\partial \boldsymbol{f}}{\partial x_{j}}=\frac{\partial}{\partial x_{j}} \boldsymbol{f}=\left(\frac{\partial f_{1}}{\partial x_{j}}, \frac{\partial f_{2}}{\partial x_{j}}, \ldots, \frac{\partial f_{n}}{\partial x_{j}}\right) .
$$

This should not be confused with the notation already used:

$$
\frac{\partial f(x)}{\partial x}:=\left(\frac{\partial f}{\partial x_{1}}, \frac{\partial f}{\partial x_{2}}, \ldots, \frac{\partial f}{\partial x_{n}}\right) .
$$

Proposition 3.8. Let $\boldsymbol{f}:=\left(f_{1}, f_{2}, \ldots, f_{n}\right)$ be a system of forms in $K[x]$, in which the components are algebraically dependent. Let $y=\left(y_{1}, \ldots, y_{n}\right)$ be a coordinate system algebraically independent of $x$. Let $\phi: K[y] \rightarrow K[x]$ be the homomorphism defined by

$$
y_{j} \mapsto f_{j},(j=1,2, \ldots, n) .
$$

Let $g=g(y) \in \operatorname{ker} \phi$ be a non-zero homogeneous polynomial of the least degree in ker $\phi$. As in Proposition [2.3, define $h_{j} \in K[x]$ by

$$
h_{j}=\frac{\partial g}{\partial y_{j}}\left(f_{1}, \ldots, f_{n}\right) .
$$

Let $\boldsymbol{h}=\left(h_{1}, \ldots, h_{n}\right)$. Then

(a) $\boldsymbol{h}$ is a syzygy of $\boldsymbol{f}$.

(b) $\boldsymbol{h}$ is a syzygy of $\partial \boldsymbol{f} / \partial x_{j}$ for every $j=1,2, \ldots, n$.

Proof. Since $g=g(y)$ is homogeneous, we have

$$
\frac{\partial g}{\partial y_{1}} y_{1}+\frac{\partial g}{\partial y_{2}} y_{2}+\cdots+\frac{\partial g}{\partial y_{n}} y_{n}=(\operatorname{deg} g) g
$$

Make the substitution $y_{i} \mapsto f_{i}$. Then we have

$$
h_{1} f_{1}+h_{2} f_{2}+\cdots+h_{n} f_{n}=0 .
$$

This shows the first assertion. By definition of $g=g(y)$, we have $g\left(f_{1}, f_{2}, \ldots, f_{n}\right)=0$. Apply the operator $\frac{\partial}{\partial x_{j}}$ to the this equality. Then we have

$$
0=\frac{\partial g\left(f_{1}, f_{2}, \ldots, f_{n}\right)}{\partial x_{j}}=\sum_{k=1}^{n} \frac{\partial g}{\partial y_{k}}\left(f_{1}, \ldots, f_{n}\right) \frac{\partial f_{k}}{\partial x_{j}}=\sum_{k=1}^{n} h_{k} \frac{\partial f_{k}}{\partial x_{j}}
$$

This shows the second assertion. 
Theorem 3.9. Let $f=f(x) \in K[x]$ be a homogeneous polynomial and put $f_{j}=\frac{\partial f}{\partial x_{j}}$. Assume that $f_{1}, \ldots, f_{n}$ are algebraically dependent. Let $\boldsymbol{f}=\left(f_{1}, \ldots, f_{n}\right)$ and let $\boldsymbol{h}=$ $\left(h_{1}, \ldots, h_{n}\right)$ be a system of forms as defined in Proposition 3.8 for $\boldsymbol{f}$. Then

(a) $f(x) \in \operatorname{Sol}(\boldsymbol{h} ; K[x])$.

(b) $f_{j}(x) \in \operatorname{Sol}(\boldsymbol{h} ; K[x])$ for $j=1,2, \ldots, n$.

(c) $f(x) \in \operatorname{Sol}\left(\partial \boldsymbol{h} / \partial x_{j} ; K[x]\right)$ for $j=1,2, \ldots, n$.

(d) $\boldsymbol{h}$ is a self-vanishing system of forms.

Proof. The assertion (a) follows immediately from Proposition 3.8 (a). Proposition 3.8 (b) says that $\boldsymbol{h}$ is a syzygy of $\partial \boldsymbol{f} / \partial x_{j}$. This means that

$$
\sum_{k=1}^{n} h_{k} \frac{\partial f_{k}}{\partial x_{j}}=0
$$

But $f_{k}=\frac{\partial f}{\partial x_{k}}$. Hence we have

$$
\sum_{k=1}^{n} h_{k} \frac{\partial f_{k}}{\partial x_{j}}=\sum_{k=1}^{n} h_{k} \frac{\partial f_{j}}{\partial x_{k}}=0
$$

This shows assertion (b).

Again by Proposition 3.8 (a) we have $\boldsymbol{h} \cdot \boldsymbol{f}=0$. For each $j$, we have

$$
\frac{\partial}{\partial x_{j}}(\boldsymbol{h} \cdot \boldsymbol{f})=\frac{\partial \boldsymbol{h}}{\partial x_{j}} \cdot \boldsymbol{f}+\boldsymbol{h} \cdot \frac{\partial \boldsymbol{f}}{\partial x_{j}}=0
$$

Again by Proposition 3.8 (b) we have $\boldsymbol{h} \cdot \partial \boldsymbol{f} / \partial x_{j}=0$. Hence $\partial \boldsymbol{h} / \partial x_{j} \cdot \boldsymbol{f}=0$. This shows that $f(x) \in \operatorname{Sol}\left(\frac{\partial}{\partial x_{j}} \boldsymbol{h} ; K[x]\right)$. Thus (c) is proved.

Since $\operatorname{Sol}(\boldsymbol{h} ; K[x])$ is a commutative ring, we have

$$
K\left[f_{1}, \ldots, f_{n}\right] \subset \operatorname{Sol}(\boldsymbol{h} ; K[x]) .
$$

Since $h_{j}(x)$ are polynomials in $f_{1}, \ldots, f_{n}$, this shows that $h_{j} \in \operatorname{Sol}(\boldsymbol{h} ; K[x])$. Thus (d) is proved.

Proposition 3.10. Let $K[x]=K\left[x_{1}, \ldots, x_{n}\right]$ be the polynomial ring and let $\boldsymbol{h}$ be a selfvanishing system of forms in $K[x]$. Then, for any $f(x) \in K[x]$, we have

$$
\mathcal{D}_{x}(\boldsymbol{h})\left(f^{(i)}(x, \boldsymbol{h})\right)=(i+1) f^{(i+1)}(x, \boldsymbol{h}) .
$$

Proof. Let $y=\left(y_{1}, y_{2}, \ldots y_{n}\right)$ be a coordinate system independent of $x$. Note that

$$
\frac{\partial f^{(i)}(x, \boldsymbol{h})}{\partial x_{j}}=\left.\left\{\frac{\partial f^{(i)}(x, y)}{\partial x_{j}}\right\}\right|_{y \mapsto \boldsymbol{h}}+\left.\left\{\sum_{k=1}^{n} \frac{\partial f^{(i)}(x, y)}{\partial y_{k}} \frac{\partial h_{k}}{\partial x_{j}}\right\}\right|_{y \mapsto \boldsymbol{h}},
$$


so $\mathcal{D}_{x}(\boldsymbol{h}) f^{(i)}(x, \boldsymbol{h})$ is equal to

$$
\left.\sum_{j=1}^{n} h_{j}(x)\left\{\frac{\partial f^{(i)}(x, y)}{\partial x_{j}}\right\}\right|_{y \mapsto \boldsymbol{h}}+\left.\sum_{j=1}^{n} h_{j}(x)\left\{\sum_{k=1}^{n} \frac{\partial f^{(i)}(x, y)}{\partial y_{k}} \frac{\partial h_{k}(x)}{\partial x_{j}}\right\}\right|_{y \mapsto \boldsymbol{h}},
$$

where $y \mapsto \boldsymbol{h}$ means substitution. Since $\mathcal{D}_{x}(\boldsymbol{h}) h_{k}(x)=\sum_{j=1}^{n} h_{j}(x) \frac{\partial h_{k}(x)}{\partial x_{j}}=0$ for every $k=1,2, \ldots, n$, the second summand of formula (2) vanishes. The first summand of formula (2) is equal to

$$
\left.\left\{\sum_{j=1}^{n} y_{j} \frac{\partial f^{(i)}(x, y)}{\partial x_{j}}\right\}\right|_{y \mapsto \boldsymbol{h}}=\left.\left\{\mathcal{D}_{x}(y) f^{(i)}(x, y)\right\}\right|_{y \mapsto \boldsymbol{h}},
$$

So

$$
\mathcal{D}_{x}(\boldsymbol{h}) f^{(i)}(x, \boldsymbol{h})=\left.\left\{\mathcal{D}_{x}(y) f^{(i)}(x, y)\right\}\right|_{y \mapsto \boldsymbol{h}}=(i+1) f^{(i+1)}(x, \boldsymbol{h})
$$

by definition of $f^{(j)}(x, y)$.

Theorem 3.11. Suppose that $\boldsymbol{h}=\left(h_{1}, \ldots, h_{n}\right)$ is a self-vanishing system of forms in $K[x]$. Then, for a homogeneous polynomial $f(x) \in K[x]$, the following conditions are equivalent:

(a) $f(x) \in \operatorname{Sol}(\boldsymbol{h} ; K[x])$.

(b) $f^{(j)}(x, \boldsymbol{h})=0$, for $j=1,2, \ldots$

(c) $f(x+\operatorname{th}(x))=f(x)$ for any $t \in K^{\prime}$, where $K^{\prime}$ is any extension field of $K$.

Proof. $(a) \Leftrightarrow(b)$ By Proposition $[3.10$, it is enough to show the case for $j=1$.

$$
\begin{aligned}
f^{(1)}(x, \boldsymbol{h}) & =\left.\left\{\mathcal{D}_{x}(y) f(x)\right\}\right|_{y \mapsto \boldsymbol{h}} \\
& =\mathcal{D}_{x}(\boldsymbol{h}) f(x) \\
& =h_{1} \frac{\partial f}{\partial x_{1}}+h_{2} \frac{\partial f}{\partial x_{2}}+\cdots+h_{n} \frac{\partial f}{\partial x_{n}}=0 .
\end{aligned}
$$

The equivalence of $(b)$ and $(c)$ follows immediately from Proposition 3.2 .

Corollary 3.12. Let $\boldsymbol{h}$ be a self-vanishing system in $K[x]$. If $f(x), g(x) \in K[x] \backslash\{0\}$, and if $f(x) g(x) \in \operatorname{Sol}(\boldsymbol{h} ; K[x])$, then both $f(x), g(x) \in \operatorname{Sol}(\boldsymbol{h} ; K[x])$.

Proof. Using Taylor expansion (Proposition [3.2), we have

$$
f(x+t \boldsymbol{h}) g(x+t \boldsymbol{h})=\sum_{j=0}^{\infty} \sum_{k+l=j} t^{k+l} f^{(k)}(x, \boldsymbol{h}) g^{(l)}(x, \boldsymbol{h}) .
$$

Let $k_{0}$ be the highest degree for which $f^{\left(k_{0}\right)} \neq 0$, and similarly $l_{0}$ for $g^{\left(l_{0}\right)}(x, \boldsymbol{h})$. Then by Theorem 3.11, $f(x) g(x) \in \operatorname{Sol}(\boldsymbol{h} ; R)$ implies $k_{0}+l_{0}=0$. Hence $k_{0}=l_{0}=0$. Again by Theorem 3.11, proof is complete. 
Corollary 3.13. Suppose that $\boldsymbol{h}=\left(h_{1}, \ldots, h_{n}\right)$ is a self-vanishing system in $R=K[x]$ such that $h_{n}(x) \neq 0$. Put

$$
s_{i}(x)=x_{i}-\frac{h_{i}(x)}{h_{n}(x)} x_{n}, \quad(1 \leq i \leq n)
$$

Let $f(x) \in \operatorname{Sol}(\boldsymbol{h} ; R)$. Then

$$
f(x)=f\left(s_{1}, s_{2}, \ldots, s_{n-1}, 0\right),
$$

and

$$
\operatorname{Sol}(\boldsymbol{h} ; K[x])=K\left[s_{1}, s_{2}, \ldots, s_{n-1}\right] \cap K[x] .
$$

Proof. It is easy to check by direct computation that

$$
\mathcal{D}_{x}(\boldsymbol{h})\left(\frac{1}{h_{n}(x)} \operatorname{det}\left(\begin{array}{cc}
x_{i} & x_{n} \\
h_{i}(x) & h_{n}(x)
\end{array}\right)\right)=0 .
$$

Hence $\mathcal{D}_{x}(\boldsymbol{h}) s_{j}(x)=0$. This shows that

$$
K\left[s_{1}, s_{2}, \ldots, s_{n-1}\right] \cap K[x] \subset \operatorname{Sol}(\boldsymbol{h} ; K[x]) .
$$

To show the converse, let $f(x) \in \operatorname{Sol}(\boldsymbol{h} ; K[x])$. Then $f(x)=f(x+$ th $)$ for an indeterminate $t$ by Theorem 3.11. Replace $t$ for $t=-\frac{x_{n}}{h_{n}(x)}$. Then we get

$$
f(x)=f\left(s_{1}, s_{2}, \ldots, s_{n-1}, 0\right) .
$$

Corollary 3.14. Let $\boldsymbol{h}=\left(h_{1}, \ldots, h_{n}\right)$ be a self-vanishing system of polynomials. Let $f(x) \in \operatorname{Sol}(\boldsymbol{h} ; K[x])$. Suppose that $f(x)$ is homogeneous of positive degree $d$. Then we have

$$
f\left(h_{1}, h_{2}, \ldots, h_{n}\right)=0 .
$$

In particular, if each $h_{j}$ has positive degree, then

$$
h_{j}\left(h_{1}, h_{2}, \ldots, h_{n}\right)=0 \text { for all } j=1,2, \ldots, n \text {. }
$$

Proof. Let $y=\left(y_{1}, \ldots, y_{n}\right)$ be a coordinate system independent of $x$, and put $\mathcal{D}_{x}(y)^{j} f(x)=$ $\frac{1}{j !} f^{(j)}(x, y)$. We have shown that $f^{(d)}(x, y)=f(y)$. In this equation substitute $y$ for $\boldsymbol{h}$. Then we have $f^{(d)}(x, \boldsymbol{h})=f(\boldsymbol{h})$. In Theorem 3.11, we showed that $f^{(j)}(x, \boldsymbol{h})=0$ for $j>0$. Thus we have $f(\boldsymbol{h})=0$. 


\section{Forms with zero Hessian and reduced self-vanishing systems}

Proposition 4.1. Let $R=K\left[x_{1}, \ldots, x_{n}\right]$. Let $\boldsymbol{f}=\left(f_{1}, f_{2}, \ldots, f_{n}\right)$ be a system of forms in $R$. Then $\operatorname{rank}\left(\frac{\partial f_{i}}{\partial x_{j}}\right)=\operatorname{tr} . \operatorname{deg}_{K} K\left(f_{1}, f_{2}, \ldots, f_{n}\right)$. In particular the following conditions are equivalent.

(1) $f_{i_{1}}, f_{i_{2}}, \ldots, f_{i_{r}}$ are algebraically dependent for every $i_{1}, i_{2}, \ldots, i_{r} \in\{1,2, \ldots, n\}$.

(2) The rank of Jacobian matrix $\left(\frac{\partial f_{i}}{\partial x_{j}}\right)$ is $<r$.

(3) tr. $\operatorname{deg}_{K} K\left(f_{i_{1}}, f_{i_{2}}, \ldots, f_{i_{r}}\right)<r$ for every $i_{1}, i_{2}, \ldots, i_{r} \in\{1,2, \ldots, n\}$.

Proof. Left to the reader.

We say that a homogeneous polynomial $f \in R=K\left[x_{1}, \ldots, x_{n}\right]$ has zero Hessian if $\operatorname{det}\left(\frac{\partial^{2} f}{\partial x_{i} \partial x_{j}}\right)=0$. (Whenever we discuss forms $f$ with zero Hessian, we assume $\operatorname{deg} f \geq 2$.) Let $f$ be a homogeneous form in $K\left[x_{1}, \ldots, x_{n}\right]$. Since the Hessian determinant of $f$ is the Jacobian determinant of the partial derivatives of $f$, the following proposition follows immediately from Lemma 4.1.

Proposition 4.2. A homogeneous polynomial $f \in R$ is a form with zero Hessian if and only if the partial derivatives

$$
\frac{\partial f}{\partial x_{1}}, \frac{\partial f}{\partial x_{2}}, \ldots, \frac{\partial f}{\partial x_{n}}
$$

are algebraically dependent.

Definition 4.3. Let $y=\left(y_{1}, \ldots, y_{n}\right)$ be a coordinate system independent of $x$. Let $f \in K[x]$ be a form with zero Hessian, and let

$$
\phi: K\left[y_{1}, \ldots, y_{n}\right] \rightarrow K\left[x_{1}, \ldots, x_{n}\right]
$$

be the homomorphism defined by $\phi\left(y_{j}\right)=\frac{\partial f}{\partial x_{j}}$. We denote by $\mathcal{I}(f)$ the kernel of $\phi$. (By Proposition 4.2, $\mathcal{I}(f) \neq 0$.)

Definition 4.4. Let $f \in R=K[x]$ be a form with zero Hessian. Let $\mathcal{I}(f) \subset K[y]$ be as in Definition 4.3. Put $f_{j}=\frac{\partial f}{\partial x_{j}}$. Let $g(y)=g\left(y_{1}, \ldots, y_{n}\right) \in \mathcal{I}(f)$ be a homogeneous form of the least degree in $\mathcal{I}(f)$. Let

$$
\begin{gathered}
h_{i}^{\prime}\left(x_{1}, x_{2}, \ldots, x_{n}\right)=\frac{\partial g}{\partial y_{i}}\left(f_{1}, \ldots, f_{n}\right), \\
h_{i}\left(x_{1}, x_{2}, \ldots, x_{n}\right)=\frac{1}{\operatorname{GCD}\left(h_{1}^{\prime}, h_{2}^{\prime}, \ldots, h_{n}^{\prime}\right)} h_{i}^{\prime}\left(x_{1}, \ldots, x_{n}\right) .
\end{gathered}
$$

We call the vector $\boldsymbol{h}^{\prime}:=\left(h_{1}^{\prime}, h_{2}^{\prime}, \ldots, h_{n}^{\prime}\right)$ a system of polynomials arising from $f(x)$, and $\boldsymbol{h}:=\left(h_{1}, \ldots, h_{n}\right)$ a reduced system of polynomials arising from $f(x)$. 
Remark 4.5. By Remark 2.4, $\boldsymbol{h} \neq 0$ as well as $\boldsymbol{h}^{\prime} \neq 0$.

Remark 4.6. Assume that $\operatorname{rank}\left(\frac{\partial^{2} f}{\partial x_{i} \partial x_{j}}\right)=n-1$. Then the ideal $\mathcal{I}(f)$ is a principal ideal of $K[y]$. In this case $g \in \mathcal{I}(f) \backslash\{0\}$ with the smallest degree is uniquely determined (up to a constant multiple). Hence $\boldsymbol{h}$, in Definition 4.4, is uniquely determined by $f$ (up to a nonzero element of $K$ ). On the other hand by Proposition 3.8 (b), we see that $\boldsymbol{h}$ is a null vector of the matrix $\left(\frac{\partial^{2} f}{\partial x_{i} \partial x_{j}}\right)$. Such a polynomial vector is unique up to a multiple of a polynomial. Hence, for any system $\boldsymbol{h}$ of forms, we can show that $\boldsymbol{h}$ is the self-vanishing system as defined in Definition 4.4 if and only if the following two conditions are satisfied.

(1) $\left(h_{1}, h_{2}, \ldots, h_{n}\right)\left(\frac{\partial^{2} f}{\partial x_{i} \partial x_{j}}\right)=0$,

(2) $\operatorname{GCD}\left(h_{1}, h_{2} \ldots, h_{n}\right)=1$.

Theorem 4.7 (Gordan-Noether). Suppose that $f(x) \in K\left[x_{1}, \ldots, x_{n}\right]$ is a form with zero Hessian. Then a variable can be eliminated from $f$ and its partial derivatives simultaneously by means of a birational transformation of the variables.

Proof. Let $\boldsymbol{h}$ be a reduced self-vanishing system arising from $f$. Then we have $f(x) \in$ $\operatorname{Sol}(\boldsymbol{h} ; K[x])$ and $\partial f(x) / \partial x_{j} \in \operatorname{Sol}(\boldsymbol{h} ; K[x])$ for $j=1,2, \ldots, n$ by Theorem 3.9 (a) and (b) respectively. By Lemma 2.1 and Remark 2.4 we may assume that $h_{n} \neq 0$. Put $s_{j}=x_{j}-\frac{h_{j}}{h_{n}} x_{n}$, for $j=1, \ldots, n-1$. Set $s_{n}=0$. Then by Corollary 3.13, $f$ is a polynomial in $s_{1}, \ldots, s_{n-1}$, and so are $\partial f(x) / \partial x_{j}$ for $j=1,2, \ldots, n$. We claim that

$$
K\left(s_{1}, \ldots, s_{n-1}, x_{n}\right)=K\left(x_{1}, \ldots, x_{n}\right) .
$$

In fact we have

$$
x_{j}=s_{j}+\frac{h_{j}(x)}{h_{n}(x)} x_{n}, \quad j=1,2, \ldots, n-1 .
$$

Since $h_{j}(x) \in \operatorname{Sol}(\boldsymbol{h} ; K[x])$, we have $h_{j}(x+t \boldsymbol{h}(x))=h_{j}(x)$ for any $t$ in any extension field of $K$ by Theorem 3.11 (c). Now let $t=-\frac{x_{n}}{h_{n}}$. Then $h_{j}(s)=h_{j}(x)$. This shows that $x_{j} \in K\left(s_{1}, \ldots, s_{n-1}, x_{n}\right)$ for all $j$, as desired.

Proposition 4.8. Suppose that $f(x) \in K[x]$ is a form with zero Hessian. Let $\mathcal{I}(f(x))$ be the ideal of $K[y]$ as defined in Definition 4.3. Then the following conditions are equivalent.

(a) The ideal $\mathcal{I}(f(x))$ contains a linear form.

(b) The partial derivatives of $f(x)$ are linearly dependent.

(c) A variable can be eliminated from $f(x)$ by means of a linear transformation of the variables.

Proof. The equivalence of (a) and (b) is clear. Suppose that there exists a non-trivial relation

$$
a_{1} f_{1}+a_{2} f_{2}+\cdots+a_{n} f_{n}=0
$$


where $f_{j}=\frac{\partial f}{\partial x_{j}}$ and $a_{j} \in K$. It is possible to choose a set of linearly independent linear forms $y_{1}, \ldots, y_{n}$ in $x_{1}, \ldots, x_{n}$ such that $\frac{\partial x_{j}}{\partial y_{1}}=a_{j}$. Then

$$
\frac{\partial f}{\partial y_{1}}=\frac{\partial f}{\partial x_{1}} \frac{\partial x_{1}}{\partial y_{1}}+\frac{\partial f}{\partial x_{2}} \frac{\partial x_{2}}{\partial y_{1}}+\cdots+\frac{\partial f}{\partial x_{n}} \frac{\partial x_{n}}{\partial y_{1}}=0 .
$$

This shows that if $f$ is expressed in terms of $y_{j}$, then $f$ does not contain $y_{1}$. Thus (b) $\Rightarrow$ (c). The same argument shows $(\mathrm{b}) \Leftarrow(\mathrm{c})$ as well.

Theorem 4.9. Let $f(x) \in K[x]$ be a form with zero Hessian and let $\boldsymbol{h}^{\prime}=\left(h_{1}^{\prime}, \ldots, h_{n}^{\prime}\right)$ be a system of polynomials associated to an element $g=g\left(y_{1}, \ldots, y_{n}\right) \in \mathcal{I}(f(x))$ of the least degree. Similarly let $\boldsymbol{h}=\left(h_{1}, \ldots, h_{n}\right)$ be the reduced system of polynomials defined by

$$
\boldsymbol{h}=\frac{1}{\operatorname{GCD}\left(\boldsymbol{h}^{\prime}\right)} \boldsymbol{h}^{\prime}
$$

(See Definition 4.4.) Then we have:

(a) $\boldsymbol{h}$ and $\boldsymbol{h}^{\prime}$ are self-vanishing systems of polynomials.

(b) $f(x) \in \operatorname{Sol}(\boldsymbol{h} ; K[x])$.

(c) $\frac{\partial}{\partial x_{j}} f(x) \in \operatorname{Sol}(\boldsymbol{h} ; K[x])$, for all $j=1,2, \ldots, n$.

Proof. (a) By Theorem [3.9, $\boldsymbol{h}^{\prime}(x)$ is a self-vanishing system. Note that $\operatorname{Sol}\left(\boldsymbol{h}^{\prime} ; K[x]\right)=$ $\operatorname{Sol}(\boldsymbol{h} ; K[x])$. By Corollary $3.12, \boldsymbol{h}(x)$ is a self-vanishing system. (b) and (c) are proved in Theorem 3.9.

Example 4.10. If $f=\left(x_{1}^{2} x_{3}+2 x_{1} x_{2} x_{4}+x_{2}^{2} x_{5}\right)\left(x_{1}^{2} x_{4}+2 x_{1} x_{2} x_{5}+x_{2}^{2} x_{6}\right)$, then $\mathcal{I}(f)$ is principal, and

$$
\boldsymbol{h}=\left(0,0, x_{2}^{3},-x_{2}^{2} x_{1}, x_{1}^{2} x_{2},-x_{1}^{3}\right),
$$

is the unique reduced self-vanishing system arising from $f$, which is as in Example 3.6.

But if $f=\left(x_{3} x_{1}+x_{4} x_{2}\right)\left(x_{5} x_{1}+x_{6} x_{2}\right)$, then $\mathcal{I}(f)$ is principal as well, and

$$
\boldsymbol{h}=\left(0,0,\left(x_{1} x_{3}+x_{2} x_{4}\right) x_{2},-\left(x_{1} x_{3}+x_{2} x_{4}\right) x_{1},-x_{2}\left(x_{1} x_{5}+x_{2} x_{6}\right), x_{1}\left(x_{1} x_{5}+x_{2} x_{6}\right)\right),
$$

is the unique reduced self-vanishing system arising from $f$, which is not as in Example 3.6.

\section{$5 \quad$ Binary and ternary forms with zero Hessian}

Theorem 5.1. Assume that $n=2$ and let $f \in K\left[x_{1}, x_{2}\right]$ be a form of degree $d$ with zero Hessian. Then $f=\left(a_{1} x_{1}+a_{2} x_{2}\right)^{d}$ for some $a_{1}, a_{2} \in K$.

Proof. Let $\mathcal{I}(f) \subset K\left[y_{1}, y_{2}\right]$ be the ideal defined in Definition 4.3. Since $\mathcal{I}(f)$ is a prime ideal, it is a principal ideal generated by a linear form. Hence the assertion follows from Proposition 4.8 . 
Lemma 5.2. Let $\boldsymbol{h}$ be a homogeneous self-vanishing system in dimension $n$. Then

$$
\operatorname{rank}\left(\frac{\partial h_{i}}{\partial x_{j}}\right)=\operatorname{tr} . \operatorname{deg}_{K} K(\boldsymbol{h})=\operatorname{Krull} \operatorname{dim} K[\boldsymbol{h}] \leq n-1
$$

and if $n \geq 3$ in addition, then

$$
\operatorname{rank}\left(\frac{\partial h_{i}}{\partial x_{j}}\right)=\operatorname{tr} \cdot \operatorname{deg}_{K} K(\boldsymbol{h})=\text { Krull } \operatorname{dim} K[\boldsymbol{h}] \leq n-2
$$

Proof. The case where $\boldsymbol{h}=0$ is trivial, so let us assume without loss of generality that $h_{1} \neq 0$. From Theorem 3.11 (c), it follows that $h_{1}(x+t \boldsymbol{h}(x))=h_{1}(x)$. If we look at the leading coefficient with respect to $t$, we see that $h_{1}(\boldsymbol{h})=0$. So

$$
\operatorname{rank}\left(\frac{\partial h_{i}}{\partial x_{j}}\right)=\operatorname{tr} . \operatorname{deg}_{K} K(\boldsymbol{h}) \leq n-1 .
$$

This gives the case $n \leq 2$, so assume from now on that $n \geq 3$.

Let $f$ be an irreducible factor of $h_{1}$. If each $h_{i}$ is a $K$-multiple of a power of $f$, then the components of $\boldsymbol{h}$ are linearly dependent in pairs, and

$$
\operatorname{rank}\left(\frac{\partial h_{i}}{\partial x_{j}}\right)=\operatorname{tr} . \operatorname{deg}_{K} K(\boldsymbol{h}) \leq 1 \leq n-2 .
$$

Otherwise, there exists an $h_{i}$ with an irreducible factor $f^{\prime}$ which is not a $K$-multiple of $f$. By Corollary [3.12, we have $f^{\prime}(x) \in \operatorname{Sol}(\boldsymbol{h} ; K[x])$ as well as $f(x) \in \operatorname{Sol}(\boldsymbol{h} ; K[x])$. From Theorem 3.11 it follows that $f(x+\operatorname{th}(x))=f^{\prime}(x+t \boldsymbol{h}(x))=0$. If we look at the leading coefficient with respect to $t$, we see that

$$
f(\boldsymbol{h}(x))=f^{\prime}(\boldsymbol{h}(x))=0,
$$

which gives the second claim of Lemma 5.2 ,

Theorem 5.3. Suppose that $f=f(x) \in K\left[x_{1}, x_{2}, x_{3}\right]$ is a form with zero Hessian. Then a variable can be eliminated from $f$ by means of a linear transformation of variables.

Proof. Let $\boldsymbol{h}=\left(h_{1}, h_{2}, h_{3}\right)$ be a reduced system of polynomials arising from $f(x)$ (See Definition 4.4). We claim that $\boldsymbol{h}$ is a constant vector. Suppose that it is not. Then Krull $\operatorname{dim} K\left[h_{1}, h_{2}, h_{3}\right]=1$ by Proposition [5.2. Thus any two of the elements $h_{1}, h_{2}, h_{3}$ has a homogeneous algebraic relation. Since $K\left[h_{1}, h_{2}, h_{3}\right]$ is an integral domain, and $K$ is algebraically closed, they should be linear relations. Thus we have $\operatorname{dim}_{K} K h_{1}+K h_{2}+$ $K h_{3}=1$. Since $\operatorname{GCD}\left(h_{1}, h_{2}, h_{3}\right)=1$, this is impossible unless they are constants. Recall that we have

$$
h_{1} \frac{\partial f}{\partial x_{1}}+h_{2} \frac{\partial f}{\partial x_{2}}+h_{3} \frac{\partial f}{\partial x_{3}}=0 .
$$

By Proposition 4.8, proof is complete. 


\section{The rational map defined by $h$}

In this section, we assume that $n \geq 4$. Let $R=K\left[x_{1}, \ldots, x_{n}\right]$ and let $\boldsymbol{h}=\left(h_{1}, \ldots, h_{n}\right)$ be any homogeneous reduced self-vanishing system. So $\operatorname{GCD}\left(h_{1}, \ldots, h_{n}\right)=1$.

Let

$$
Z: \mathbb{P}^{n-1}(x) \rightarrow \mathbb{P}^{n-1}(y)
$$

be the rational map defined by the correspondence $x=\left(x_{1}: \cdots: x_{n}\right) \mapsto\left(h_{1}: \cdots: h_{n}\right)$. Let $W$ be the image of $Z$ and $T$ the fundamental locus of $Z$ in $\mathbb{P}^{n-1}(x)$ defined by the equations $h_{1}(x)=h_{2}(x)=\cdots=h_{n}(x)=0$. The algebraic set $W \subset \mathbb{P}^{n-1}(y)$ is defined by the kernel of the homomorphism defined by

$$
y_{j} \mapsto h_{j},(j=1,2, \ldots, n) .
$$

which corresponds to $Z$.

Proposition 6.1. The following conditions are equivalent.

(a) $\operatorname{deg}\left(h_{j}\right)=0$, i.e., $\boldsymbol{h}$ is a constant vector.

(b) $\operatorname{dim} W=0$, i.e., $W$ is a one-point set.

(c) $T$ is empty, i.e., $Z$ is a morphism.

Proof. Suppose that $\boldsymbol{h}$ is not a constant. Then we have

$$
h_{j}\left(h_{1}, \ldots, h_{n}\right)=0 \text { for every } j=1, \ldots, n .
$$

by Corollary 3.14, Thus any specialization of $\left(h_{1}, \ldots, h_{n}\right)$ is a point of $T$. This shows that if $T$ is empty, then $\boldsymbol{h}$ is a constant vector. All other implications are trivial.

Proposition 6.2. If $\operatorname{dim} W \geq 1$, then

$$
2 \leq \text { Krull } \operatorname{dim} K[x] /\left(h_{1}, \ldots, h_{n}\right) \leq n-2,
$$

or equivalently,

$$
1 \leq \operatorname{dim} T \leq n-3
$$

Proof. Since $T$ is not empty, the ideal $\left(h_{1}, h_{2}, \ldots, h_{n}\right) \subset K[x]$ is not the unit ideal by the previous proposition. On the other hand, since $\operatorname{GCD}\left(h_{1}, \ldots, h_{n}\right)=1$, it is not a principal ideal. Hence $\operatorname{ht}\left(h_{1}, \ldots, h_{n}\right) \geq 2$. This shows that $\operatorname{dim} T=\operatorname{Krull} \operatorname{dim} K[x] /\left(h_{1}, \ldots, h_{n}\right)-$ $1 \leq n-3$. On the other hand there exists a surjective homomorphism of rings:

$$
\begin{gathered}
K\left[x_{1}, \ldots, x_{n}\right] /\left(h_{1}, \ldots, h_{n}\right) \rightarrow K\left[h_{1}, \ldots, h_{n}\right], \\
x_{j} \mapsto h_{j},
\end{gathered}
$$

provided that $\operatorname{deg} \boldsymbol{h}>0$. Note that $K\left[x_{1}, \ldots, x_{n}\right] /\left(h_{1}, \ldots, h_{n}\right)$ is the fiber at the origin of the inclusion map $K\left[h_{1}, \ldots, h_{n}\right] \rightarrow K\left[x_{1}, \ldots, x_{n}\right]$ and Krull $\operatorname{dim} K\left[h_{1}, \ldots, h_{n}\right] \leq n-2$ by Lemma 5.2. Hence we have Krull $\operatorname{dim} K\left[x_{1}, \ldots, x_{n}\right] /\left(h_{1}, \ldots, h_{n}\right) \geq 2$. 
For the rest of this section we assume that $\operatorname{dim} W=1$. In this case the fiber of $Z: \mathbb{P}^{n-1}(x) \rightarrow \mathbb{P}^{n-1}(y)$ is a hypersurface of $\mathbb{P}^{n-1}(x)$. Thus, for any $\omega \in \mathbb{P}^{n-1}(y), Z^{-1}(\omega)$ is defined by one homogeneous polynomial in $x=\left(x_{1}, \ldots, x_{n}\right)$.

Definition 6.3. Let $Z: \mathbb{P}^{n-1}(x) \rightarrow \mathbb{P}^{n-1}(y)$ be as above. Let $\omega=\left(\omega_{1}: \omega_{2}: \cdots\right.$ : $\left.\omega_{n}\right) \in \mathbb{P}^{n-1}(y)$. We denote by $g^{(\omega)}(x)$ the square-free polynomial in $K[x]$ that defines the hypersurface of the fiber of $Z$ at $\omega \in W \subset \mathbb{P}^{n-1}(y)$.

For each point $\omega=\left(\omega_{1}: \omega_{2}: \cdots: \omega_{n}\right) \in \mathbb{P}^{n-1}$, we define the differential operator $\mathcal{D}_{x}(\omega)$ on $R=K\left[x_{1}, \ldots, x_{n}\right]$ by

$$
\mathcal{D}_{x}(\omega) f(x)=\sum_{j=1}^{n} \omega_{j} \frac{\partial f(x)}{\partial x_{j}}
$$

whose value is determined up to a non-zero constant factor. Hence we may speak of the set of solutions of the equation $\mathcal{D}_{x}(\omega) f(x)=0$. We denote the space of solutions of $\mathcal{D}_{x}(\omega) f(x)=0$ in $R=K\left[x_{1}, \ldots, x_{n}\right]$ by $\operatorname{Sol}(\omega ; R)$. It is a subring of $R$. For any subset $U$ of $\mathbb{P}^{n-1}$ we denote by $\operatorname{Sol}(U ; R)$ the space of solutions of the system of linear differential equations

$$
\mathcal{D}_{x}(\omega)(f(x))=0, \omega \in U .
$$

If we denote by $L(U)$ the linear closure of $U$ in $\mathbb{P}^{n-1}$ it is easy to see that

$$
\operatorname{Sol}(U ; R)=\operatorname{Sol}(L(U) ; R)
$$

If $U=\left\{\omega^{(1)}, \omega^{(2)}, \ldots, \omega^{(s)}\right\}$ is a finite set, then $\operatorname{Sol}(U ; R)$ is also denoted as

$$
\operatorname{Sol}\left(\omega^{(1)}, \omega^{(2)}, \ldots, \omega^{(s)} ; R\right) .
$$

The same notation is used if we replace $\mathbb{P}^{n-1}(y)$ for the vector space $K^{n}$ in the obvious sense. Namely for a linear subspace $L$ of $K^{n}$, we denote by $\operatorname{Sol}(L ; R)$, the set of solutions of the differential equations

$$
\mathcal{D}_{x}(\boldsymbol{a}) F(x)=0 \text { for all } \boldsymbol{a} \in L,
$$

where $\boldsymbol{a}$ denotes a row vector in $L$ regarded as a system of constants. In a set theoretic notation,

$$
\operatorname{Sol}(L ; R)=\left\{F(x) \in R \mid\left(a_{1} \frac{\partial}{\partial x_{1}}+a_{2} \frac{\partial}{\partial x_{2}}+\cdots+a_{n} \frac{\partial}{\partial x_{n}}\right) F(x)=0,\left(a_{1}, \ldots, a_{n}\right) \in L\right\} .
$$

Note that $\operatorname{Sol}(L ; R)$ is a subring of $R$ generated by homogeneous linear forms.

The relation between the subring $\operatorname{Sol}(L ; R)$ of $R$ and the ideal $I$ for the linear subspace in $\mathbb{P}^{n-1}$ is very important for us. In the next theorem and corollary we describe a set of generators of the subring $\operatorname{Sol}(L ; R)$ and a set of generators that defines the linear space $L$ as a subspace of $\mathbb{P}^{n-1}$. 
Theorem 6.4. Let $R=K\left[x_{1}, \ldots, x_{n}\right]$ be the polynomial ring over $K$. Let $A=\left(a_{i}^{(j)}\right)$ be a $k \times n$ matrix, $a_{i}^{(j)} \in K$, with $\boldsymbol{a}^{(j)}=\left(a_{1}^{(j)}, a_{2}^{(j)}, \ldots, a_{n}^{(j)}\right)$ as the $j$-th row. Suppose that the rows are linearly independent. Assume that $k<n$. Then the set of solutions as a subring of $K[x]$

$$
\operatorname{Sol}\left(\boldsymbol{a}^{(1)}, \ldots, \boldsymbol{a}^{(k)} ; R\right):=\bigcap_{j=1}^{k} \operatorname{Sol}\left(\boldsymbol{a}^{(j)} ; R\right)
$$

is isomorphic to the polynomial ring in $n-k$ variables. It is generated by the $(k+1) \times(k+1)$ minors of the matrix

$$
A^{\prime}:=\left(\begin{array}{ccccc}
a_{1}^{(1)} & a_{2}^{(1)} & \cdots & a_{n-1}^{(1)} & a_{n}^{(1)} \\
a_{1}^{(2)} & a_{2}^{(2)} & \cdots & a_{n-1}^{(2)} & a_{n}^{(2)} \\
& & & & \\
a_{1}^{(k)} & a_{2}^{(k)} & \cdots & a_{n-1}^{(k)} & a_{n}^{(k)} \\
\hline x_{1} & x_{2} & \cdots & x_{n-1} & x_{n}
\end{array}\right)
$$

Proof. Let $V$ be the vector space of common syzygies of $\boldsymbol{a}^{(1)}, \ldots, \boldsymbol{a}^{(k)}$ over $K$. Take $\boldsymbol{v}=\left(v_{1}, v_{2}, \ldots, v_{n}\right) \in V$ and define a linear form

$$
l_{\boldsymbol{v}}:=v_{1} x_{1}+v_{2} x_{2}+\cdots+v_{n} x_{n}
$$

Let $k=n-\operatorname{dim} V$. Take a basis $\boldsymbol{v}_{1}, \boldsymbol{v}_{2}, \ldots, \boldsymbol{v}_{n-k}$ of $V$, and extend it to a basis $\boldsymbol{v}_{1}, \boldsymbol{v}_{2}, \ldots, \boldsymbol{v}_{n}$ of $K^{n}$. Let $l_{1}, l_{2}, \ldots, l_{n}$ be the corresponding linear forms as defined in (4). Then

$$
R=K\left[l_{1}, l_{2}, \ldots, l_{n}\right] .
$$

Now take $f \in R$. Then we can write $f=g\left(l_{1}, l_{2}, \ldots, l_{n}\right)$ where $g \in K\left[y_{1}, y_{2}, \ldots, y_{n}\right]$. Write $f_{i}=\partial f / \partial x_{i}$ and $g_{i}=\partial g / \partial y_{i}$ for $i=1,2, \ldots, n$. Then

$$
\left(f_{1}, f_{2}, \ldots, f_{n}\right)=g_{1}\left(l_{1}, l_{2}, \ldots, l_{n}\right) \boldsymbol{v}_{1}+g_{2}\left(l_{1}, l_{2}, \ldots, l_{n}\right) \boldsymbol{v}_{2}+\cdots+g_{n}\left(l_{1}, l_{2}, \ldots, l_{n}\right) \boldsymbol{v}_{n}
$$

Hence $f \in \operatorname{Sol}\left(\boldsymbol{a}^{(1)}, \ldots, \boldsymbol{a}^{(k)} ; R\right)$ if and only if $g_{n-k+1}=g_{n-k+2}=\cdots=g_{n}=0$, i.e., $f \in K\left[l_{1}, l_{2}, \ldots, l_{n-k}\right]$. Indeed, $K\left[l_{1}, l_{2}, \ldots, l_{n-k}\right]$ in isomorphic to the polynomial ring in $n-k$ variables.

If we take for $f$ a $(k+1) \times(k+1)$ minor of $A^{\prime}$, then $f\left(\boldsymbol{a}^{(j)}\right)=0$ because $f\left(\boldsymbol{a}^{(j)}\right)$ is the determinant of a matrix of which the last row coincides with row $j$. From this, we infer that the $(k+1) \times(k+1)$ minor of $A^{\prime}$ are linear combinations of $l_{1}, l_{2}, \ldots, l_{n-k}$. So it remains to show the converse, i.e., that $l \boldsymbol{v}$ is a linear combination of the $(k+1) \times(k+1)$ minors of $A^{\prime}$ for every $\boldsymbol{v} \in V$.

Suppose first that $k=n-1$. Then $V=K w_{1}$ has dimension 1 , and $l_{\boldsymbol{v}}, l_{1}$ and $\operatorname{det} A^{\prime}$ are the same up to a nonzero constant for every nonzero $\boldsymbol{v} \in V$. So $\boldsymbol{l}_{\boldsymbol{v}}$ is a linear combination of the $(k+1) \times(k+1)$ minors of $A^{\prime}$ for every $\boldsymbol{v} \in V$.

Suppose next that $k<n-1$. Then we can extend $\boldsymbol{a}^{(1)}, \ldots, \boldsymbol{a}^{(k)}$ to a basis $\boldsymbol{a}^{(1)}, \ldots, \boldsymbol{a}^{(n-1)}$ of the syzygies over $K$ of $\boldsymbol{v}$. Now the case $k=n-1$ yields $l_{\boldsymbol{v}}$ as a determinant of an $n \times n$ matrix up to a nonzero constant. If we expand this matrix along rows $k+1, k+2, \ldots, n-1$, then we get a linear combination of the $(k+1) \times(k+1)$ minors of $A^{\prime}$. 
Corollaries 6.5 and 6.6 that follow are other ways to describe the set of linear forms as generators for $\operatorname{Sol}(L ; R)$.

Corollary 6.5. Let $R$ and $A$ be the same as Theorem 6.4, and furthermore let $L$ be the vector subspace in $K^{n}$ generated by the rows of $A$. Then the set of $\operatorname{solutions} \operatorname{Sol}(L ; R)$, as a subring of $R=K\left[x_{1}, \ldots, x_{n}\right]$, is generated by the linear forms $l=l(x)$ such that

$$
l\left(a_{1}^{(j)}, a_{2}^{(j)}, \ldots, a_{n}^{(j)}\right)=0
$$

for all $j=1,2, \ldots, k$.

Proof. This follows from the proof of Theorem 6.4,

Corollary 6.6. Suppose that $U \subset \mathbb{P}^{n-1}(y)$ is a subset and let $L(U)$ be the linear closure of $U$. Then $\operatorname{Sol}(U ; R)=\operatorname{Sol}(L(U) ; R)$ is generated by the linear forms of

$$
\left\{l(x) \in K\left[x_{1}, x_{2}, \ldots, x_{n}\right] \mid l\left(\omega_{1}, \omega_{2}, \ldots, \omega_{n}\right)=0 \text { for all }\left(\omega_{1}: \omega_{2}: \cdots: \omega_{n}\right) \in L(U)\right\},
$$

as a subring of $K[x]$.

The following two theorems are very important to determine the forms in four and five variables with zero Hessian.

Theorem 6.7. Let $\boldsymbol{h}=\left(h_{1}, \ldots, h_{n}\right)$ be a self-vanishing system of forms in $R=K\left[x_{1}, \ldots, x_{n}\right]$. Let $Z: \mathbb{P}^{n-1}(x) \rightarrow \mathbb{P}^{n-1}(y)$ be the rational map defined by $y_{j}=h_{j}(x)$. Let $T \subset \mathbb{P}^{n-1}(x)$ be the fundamental locus and $W$ the image of $Z$. Assume that $\operatorname{dim} W=1$. Let

$$
i: \mathbb{P}^{n-1}(y) \rightarrow \mathbb{P}^{n-1}(x)
$$

be the natural map $y_{j} \rightarrow x_{j}$. Then $i(L(W)) \subset T$, where $L(W)$ is the linear closure of $W$ in $\mathbb{P}^{n-1}(y)$.

Proof. Recall that $T$ is defined by the polynomials $h_{1}(x), \ldots, h_{n}(x)$. On the other hand $i(L(W))$ is defined by linear forms which vanish on the set $i(L(W))$. Hence, in view of Corollary 6.6, this follows immediately from Theorem 6.8 below.

Theorem 6.8. With the same notations and assumptions as in Theorem 6.7, we have

$$
h_{j}(x) \in \operatorname{Sol}(L(W) ; R) \text { for all } j=1,2, \ldots, n \text {. }
$$

We prove it after some propositions. For the rest of this section we fix notation and assumption of Theorem 6.7. In particular it is assumed that $\operatorname{dim} W=1$.

Proposition 6.9. For any $\omega \in W$, we have $g^{(\omega)}(x) \in \operatorname{Sol}(\boldsymbol{h} ; R)$. 
Proof. Choose a hyperplane $H \subset \mathbb{P}^{n-1}(y)$ such that $\omega \in H$, and suppose that $H$ is defined by the linear equation

$$
a_{1} y_{1}+\cdots+a_{n} y_{n}=0 .
$$

Put $f(x)=\sum_{j=1}^{n} a_{j} h_{j}(x)$. Since $K$ is infinite, it is possible to choose $H$ such that $f(x) \neq 0$. Then for any $\alpha \in Z^{-1}(\omega)$, if $\alpha \in T$, then $h_{j}(\alpha)=0$ for all $j$ by definition of $T$. Hence $f(\alpha)=0$. If $\alpha \notin T$, then

$$
f(\alpha)=\sum_{j=1}^{n} a_{j} h_{j}(\alpha)=c \sum_{j=1}^{n} a_{j} \omega_{j}=0 .
$$

This shows that any point on $Z^{-1}(\omega)$ is a zero of $f(x)$. Hence $f(x)$ is a multiple of $g^{(\omega)}(x)$. By Corollary 3.12, this proves that $g^{(\omega)}(x) \in \operatorname{Sol}(\boldsymbol{h} ; R)$ for any $\omega \in W$.

Proposition 6.10. For each point $\omega \in W$, we have $g^{(\omega)}(x) \in \operatorname{Sol}(L(W) ; R)$.

Proof is preceded by two lemmas.

Lemma 6.11. For $\omega, \omega^{\prime} \in W$, the polynomial $\mathcal{D}_{x}\left(\omega^{\prime}\right) g^{(\omega)}(x)$ is divisible by $g^{\left(\omega^{\prime}\right)}(x)$.

Proof. In Proposition [6.9, we proved that $g^{(\omega)}(x) \in \operatorname{Sol}(\boldsymbol{h} ; R)$. So if $\mathcal{D}_{x}(\boldsymbol{h})$ is applied to $g^{(\omega)}(x)$, it becomes 0 . Namely,

$$
h_{1}(x) \frac{\partial g^{(\omega)}(x)}{\partial x_{1}}+\cdots+h_{n}(x) \frac{\partial g^{(\omega)}(x)}{\partial x_{n}}=0 .
$$

Choose $\alpha \in Z^{-1}\left(\omega^{\prime}\right)$ and make the substitution $x \mapsto \alpha$ in the above equality. Then the left hand side is the same as

$$
\mathcal{D}_{x}\left(\omega^{\prime}\right) g^{(\omega)}(x)
$$

evaluated at $x=\alpha$. We have shown that the zero locus of $g^{\left(\omega^{\prime}\right)}(x)$ is contained in that of $\mathcal{D}_{x}\left(\omega^{\prime}\right) g^{(\omega)}(x)$. Since $g^{\left(\omega^{\prime}\right)}(x)$ is square-free, this proves the assertion.

Lemma 6.12. For any point $\omega \in W$, we have $\mathcal{D}_{x}(\omega) g^{(\omega)}(x)=0$.

Proof. In the previous lemma let $\omega^{\prime}=\omega$. Then we have proved that $\mathcal{D}_{x}(\omega) g^{(\omega)}(x)=$ $r(x) g^{(\omega)}(x)$ for some $r(x) \in R$. For the degree reason, we get the assertion.

Proof of Proposition 6.10. By Lemma 6.12, we have

$$
\mathcal{D}_{x}(\omega)\left(\mathcal{D}_{x}\left(\omega^{\prime}\right) g^{(\omega)}(x)\right)=\mathcal{D}_{x}\left(\omega^{\prime}\right)\left(\mathcal{D}_{x}(\omega) g^{(\omega)}(x)\right)=0 .
$$

By Lemma 6.11, we may write $\mathcal{D}_{x}\left(\omega^{\prime}\right) g^{(\omega)}(x)=r(x) g^{\left(\omega^{\prime}\right)}(x)$ for some $r(x)$. Since

$$
\mathcal{D}_{x}(\omega)\left(r(x) g^{\left(\omega^{\prime}\right)}(x)\right)=0,
$$

$r(x) g^{\left(\omega^{\prime}\right)}(x) \in \operatorname{Sol}(\omega ; R)$. Therefore, since a constant vector is a self-vanishing system, we have $g^{\left(\omega^{\prime}\right)}(x) \in \operatorname{Sol}(\omega ; R)$ by Corollary 3.12 . 
Proof of Theorem 6.8. We have to show that $h_{j}(x) \in \operatorname{Sol}(L(W) ; R)$. We may assume that $h_{j} \neq 0$. Let $W_{j}$ be the intersection of $W$ and the hyperplane defined by $y_{j}=0$ in the space $\mathbb{P}^{n-1}(y)$, and let $H_{j} \subset \mathbb{P}^{n-1}(x)$ be the hypersurface defined by $h_{j}(x)=0$. Then $W_{j}$ is a finite set, since $\operatorname{dim} W=1$ and $W$ is not contained in the above hyperplane. Thus, if we write $W_{j}=\left\{\omega^{(1)}, \omega^{(2)}, \ldots, \omega^{(s)}\right\}$, then

$$
H_{j} \supset Z^{-1}\left(\omega^{(1)}\right) \cup Z^{-1}\left(\omega^{(2)}\right) \cup \cdots \cup Z^{-1}\left(\omega^{(s)}\right) .
$$

Conversely if $\alpha \in H_{j} \backslash T$, then $Z(\alpha) \in W_{j}$. Thus

$$
H_{j} \backslash T \subset Z^{-1}\left(\omega^{(1)}\right) \cup Z^{-1}\left(\omega^{(2)}\right) \cup \cdots \cup Z^{-1}\left(\omega^{(s)}\right) .
$$

Since $H_{j}$ is purely of codimension 1 and $T$ has codimension at least 2 (Proposition 6.2), it in fact shows that

$$
H_{j} \subset Z^{-1}\left(\omega^{(1)}\right) \cup Z^{-1}\left(\omega^{(2)}\right) \cup \cdots \cup Z^{-1}\left(\omega^{(s)}\right) .
$$

Thus we have proved that up to a nonzero constant factor, the square-free part of $h_{j}(x)$ is equal to

$$
g^{\left(\omega^{(1)}\right)}(x) \times g^{\left(\omega^{(2)}\right)}(x) \times \cdots \times g^{\left(\omega^{(s)}\right)}(x) .
$$

By Proposition 6.10, this completes the proof of Theorem 6.8.

\section{Quaternary and quinary forms with zero Hessian}

Theorem 7.1. Let $f=f\left(x_{1}, x_{2}, x_{3}, x_{4}\right) \in R=K\left[x_{1}, x_{2}, x_{3}, x_{4}\right]$ be a form with zero Hessian. Then $f$ can be transformed into a form with three variables via a linear transformation of the variables $x_{1}, x_{2}, x_{3}, x_{4}$.

Proof. Let $\boldsymbol{h}=\left(h_{1}, h_{2}, h_{3}, h_{4}\right)$ be a reduced self-vanishing system of forms arising from $f$. Then by Lemma 5.2, Krull $\operatorname{dim} K\left[h_{1}, h_{2}, h_{3}, h_{4}\right] \leq n-2=2$. This shows $\operatorname{dim} W \leq 1$. Put $s=\operatorname{dim} L(W)$ (as a linear variety in $\left.\mathbb{P}^{3}\right)$. Note that $s+1=\operatorname{dim}_{K}\left(K h_{1}+\cdots+K h_{4}\right)$. Since $i(L(W)) \subset T$ by Theorem 6.7 and $\operatorname{dim} T \leq 1$ by Proposition 6.2 , we have $s \leq 1$. If $s=0$, we get the result by Proposition 6.1. If $s=1$, we may assume that $h_{1}(x)=h_{2}(x)=0$ by Lemmas 2.1 and 2.2. Let $g(y) \in K\left[y_{1}, \ldots, y_{4}\right]$ be the polynomial through which $h_{1}, \ldots, h_{4}$ are defined (cf. Definition 4.4). Then $h_{1}=h_{2}=0$ implies that $\frac{\partial g}{\partial y_{1}}=\frac{\partial g}{\partial y_{2}}=0$ by the minimality of the degree. (cf. Proposition 2.3 and Remark 2.4.) Hence $g$ is a form only in two variables. Since $g$ should be irreducible, $g$ is a linear form. This implies that there exists a linear relation among the partial derivatives of $f$. Hence the assertion follows from Proposition 4.8,

Proposition 7.2. Let $f=f\left(x_{1}, x_{2}, x_{3}, x_{4}, x_{5}\right) \in R=K\left[x_{1}, x_{2}, x_{3}, x_{4}, x_{5}\right]$ be a form with zero Hessian. Let $\boldsymbol{h}=\left(h_{1}, h_{2}, h_{3}, h_{4}, h_{5}\right)$ be a self-vanishing system of polynomials associated to $f$ as defined in Definition 4.4. Assume that there exists no linear relations among the partial derivatives of $f$. Then by a linear change of variables, $f$ can be transformed into a form so that $h_{1}=h_{2}=0$, and $h_{3}, h_{4}, h_{5}$ are polynomials only in $x_{1}, x_{2}$. 
Proof. Let $Z: \mathbb{P}^{4}(x) \rightarrow \mathbb{P}^{4}(y)$ be the rational map defined by $y_{j}=h_{j}(x)$ and let $T$ be the fundamental locus and $W$ the image of $Z$. We will prove that $\operatorname{dim} W \leq 1$ in the next section. If $\operatorname{dim} W=0$, then a variable can be eliminated from $f$, since $\boldsymbol{h}$ is a constant vector by Proposition 6.1. Assume that $\operatorname{dim} W=1$. Then we have $i(L(W)) \subset T$ by Theorem 6.7. On the other hand we have $\operatorname{dim} T \leq 2$ by Proposition 6.2. This shows $\operatorname{dim} L(W) \leq 2$ as a linear subspace in $\mathbb{P}^{4}$. Let $s=\operatorname{dim} L(W)$ or equivalently $s+1=$ $\operatorname{dim}_{K} \sum_{j=1}^{5} K h_{j}$. Since $\operatorname{dim} W=1, s \geq 1$. If $s=1$, we may assume $h_{1}=h_{2}=h_{3}=0$. As in the proof of Theorem 7.1 this would imply that there exist a linear relation among the partial derivatives of $f$. Since we have excluded this case, we are left with the case $s=2$. Then we may assume that $h_{1}=h_{2}=0$ and $h_{j} \in \operatorname{Sol}(i(L(W)) ; R)$ for $j=3,4,5$. The linear subspace $i(L(W))$ consists of vectors $(0,0, *, *, *)$. Hence $h_{3}, h_{4}, h_{5}$ should be polynomials only in $x_{1}$ and $x_{2}$.

Theorem 7.3. Let $R=K\left[x_{1}, x_{2}, x_{3}, x_{4}, x_{5}\right]$, and let $\Delta$ be a homogeneous polynomial of the form

$$
\Delta=p_{3}\left(x_{1}, x_{2}\right) x_{3}+p_{4}\left(x_{1}, x_{2}\right) x_{4}+p_{5}\left(x_{1}, x_{2}\right) x_{5}
$$

Then any element in the algebra $K\left[x_{1}, x_{2}\right][\Delta]$ is a polynomial with zero Hessian.

Conversely, let $f$ be a homogeneous form in five variables with zero Hessian and assume that $f$ properly involves five variables. Then we can choose $\Delta$ such that $f$ can be transformed into a homogeneous polynomial in the algebra $K\left[x_{1}, x_{2}\right][\Delta]$ by means of a linear change of variables.

Proof. Put $\boldsymbol{f}=\left(f_{1}, \ldots, f_{5}\right)$, where $f_{j}=\frac{\partial f}{\partial x_{j}}$. Suppose first that $f \in K\left[x_{1}, x_{2}\right][\Delta]$. Assume without loss of generality that $f_{5} \neq 0$. Then $f_{3} / f_{5}=p_{3} / p_{5} \in K\left(x_{1} / x_{2}\right)$ and $f_{4} / f_{5}=$ $p_{4} / p_{5} \in K\left(x_{1} / x_{2}\right)$, so $f_{3}, f_{4}, f_{5} \in K\left(x_{1} / x_{2}, f_{5}\right)$. Hence tr. $\operatorname{deg}_{K} K\left(f_{3}, f_{4}, f_{5}\right) \leq 2$ and tr. $\operatorname{deg}_{K} K\left(f_{1}, f_{2}, f_{3}, f_{4}, f_{5}\right) \leq 4$. On account of Proposition 4.1, $f$ has zero Hessian.

Suppose next that $f$ has zero Hessian. On account of Proposition $4.2, f_{1}, f_{2}, f_{3}, f_{4}, f_{5}$ are algebraically dependent over $K$. Put $\boldsymbol{f}=\left(f_{1}, \ldots, f_{5}\right)$, where $f_{j}=\frac{\partial f}{\partial x_{j}}$. Let $\boldsymbol{h}=$ $\left(h_{1}, \ldots, h_{5}\right)$ be a reduced self-vanishing system arising from $f(x)$. We have proved that $\boldsymbol{h} \cdot \frac{\partial}{\partial x_{j}} \boldsymbol{f}=0, j=1, \ldots, 5$ in Corollary 3.9. This shows that $f(x) \in \operatorname{Sol}\left(\frac{\partial}{\partial x_{1}} \boldsymbol{h}, \ldots, \frac{\partial}{\partial x_{5}} \boldsymbol{h} ; R\right)$. By Proposition 7.2, we may assume that $h_{1}=h_{2}=0$ and $h_{3}, h_{4}, h_{5}$ involve only $x_{1}, x_{2}$. Let $\tilde{K}$ be the algebraic closure of $K\left(x_{1}, x_{2}\right)$. It follows from Theorem 6.4 that $f$ is a polynomial over $\tilde{K}$ in

$$
\tilde{A}^{\prime}:=\left|\begin{array}{ccc}
\frac{\partial h_{3}}{\partial x_{1}} & \frac{\partial h_{4}}{\partial x_{1}} & \frac{\partial h_{5}}{\partial x_{1}} \\
\frac{\partial h_{3}}{\partial x_{2}} & \frac{\partial h_{4}}{\partial x_{2}} & \frac{\partial h_{5}}{\partial x_{2}} \\
\hline x_{3} & x_{4} & x_{5}
\end{array}\right| .
$$

Notice that $\tilde{A}^{\prime} \in R$ has only one irreducible factor which is not contained in $K\left[x_{1}, x_{2}\right]$. Furthermore, we can choose $\Delta$ to be this factor. Then $f \in \tilde{K}[\delta]$, say

$$
f=b_{0}+b_{1} \Delta+b_{2} \Delta^{2}+\cdots .
$$

Then the coefficient of $x_{j}^{i}$ of $f$ as a polynomial in $K\left[x_{1}, x_{2}\right]\left[x_{3}, x_{4}, x_{5}\right]$ equals $b_{i} p_{j}^{i}$, for $j=3,4,5$. So $b_{i} \in K\left(x_{1}, x_{2}\right)$. Since $\operatorname{GCD}\left(p_{3}^{i}, p_{4}^{i}, p_{5}^{i}\right)=1$, we infer that $b_{i} \in K\left[x_{1}, x_{2}\right]$. This holds for all $i$, so $f \in K\left[x_{1}, x_{2}\right][\Delta]$. 
If $\Delta$ is cubic, then we can take $\Delta=A^{\prime}$. But if $\Delta$ has larger degree, then this is not always possible. Take e.g. $\Delta$ irreducible of even degree, then we cannot take $\Delta=A^{\prime}$, because $A^{\prime}$ has always odd degree. It is however possible to write $\Delta$ in the same way as $A^{\prime}$, but with the $h_{i}^{(j)}$ replaced by other polynomials in $K\left[x_{1}, x_{2}\right]$.

Theorem 7.4. Let $n \geq 4$. Let $\Delta \in K\left[x_{1}, x_{2}, \ldots, x_{n}\right]$ be a homogeneous polynomial of degree at least 2 of the form

$$
\Delta=p_{3}\left(x_{1}, x_{2}\right) x_{3}+p_{4}\left(x_{1}, x_{2}\right) x_{4}+\cdots+p_{n}\left(x_{1}, x_{2}\right) x_{n}
$$

Then there are polynomials $a_{i}^{(j)}$ in $K\left[x_{1}, x_{2}\right]$, such that $\boldsymbol{a}^{(j)}=\left(a_{3}^{(j)}, a_{4}^{(j)}, \ldots, a_{n}^{(j)}\right)$ is homogeneous for all $3 \leq j \leq n-1$, and

$$
\Delta=\left|\begin{array}{cccc}
a_{3}^{(3)} & a_{4}^{(3)} & \cdots & a_{n}^{(3)} \\
a_{3}^{(4)} & a_{4}^{(4)} & \cdots & a_{n}^{(4)} \\
\vdots & \vdots & & \vdots \\
a_{3}^{(n-1)} & a_{4}^{(n-1)} & \cdots & a_{n}^{(n-1)} \\
\hline x_{3} & x_{4} & \cdots & x_{n}
\end{array}\right| .
$$

Proof. If theorem 7.4 holds for $\Delta\left(x_{1}, x_{2}, A\left(x_{3}, x_{4}, \ldots, x_{n}\right)\right)$ for some $A \in \mathrm{GL}(n-2, K)$ instead of $\Delta$ itself, then we can substitute $\left(x_{3}, x_{4}, \ldots, x_{n}\right)$ by $A^{-1}\left(x_{3}, x_{4}, \ldots, x_{n}\right)$ in the matrix with determinant $\Delta\left(x_{1}, x_{2}, A\left(x_{3}, x_{4}, \ldots, x_{n}\right)\right)$, to obtain a matrix with determinant $\Delta$. Without affecting its determinant, we can get the last matrix of the form of theorem 7.4 by way of column operations and multiplying the first row with a nonzero constant. This allows us to replace $\left(p_{3}\left(x_{1}, x_{2}\right), p_{4}\left(x_{1}, x_{2}\right), \ldots, p_{n}\left(x_{1}, x_{2}\right)\right)$ by $\left(p_{3}\left(x_{1}, x_{2}\right), \ldots, p_{n}\left(x_{1}, x_{2}\right)\right) A$ for any $A \in \mathrm{GL}(n-2, K)$.

Suppose first that $p_{3}\left(x_{1}, x_{2}\right), p_{4}\left(x_{1}, x_{2}\right), \ldots, p_{n}\left(x_{1}, x_{2}\right)$ are linearly dependent over $K$. Then we may assume that $p_{n}\left(x_{1}, x_{2}\right)=0$. If $n=4$, then we take $a_{3}^{(3)}=0$ and $a_{4}^{(3)}=$ $-p_{3}\left(x_{1}, x_{2}\right)$. So assume that $n \geq 5$. Then we take $a_{i}^{(n-1)}=0$ for all $3 \leq i<n$ and $a_{n}^{(n-1)}=-1$. Furthermore, we take $a_{n}^{(j)}=0$ for all $3 \leq j<n-1$. By induction on $n$, there exist $a_{i}^{(j)}$ as claimed.

Suppose next that $p_{3}\left(x_{1}, x_{2}\right), p_{4}\left(x_{1}, x_{2}\right), \ldots, p_{n}\left(x_{1}, x_{2}\right)$ are linearly independent over $K$. Take $\alpha_{3} \in K$, such that $p_{3}\left(\alpha_{3}, 1\right) \neq 0$, and assume without loss of generality that $p_{i}\left(\alpha_{3}, 1\right)=0$ for all $i \neq 3$. Take $\alpha_{4} \in K$, such that $p_{4}\left(\alpha_{4}, 1\right) \neq 0$, and assume without loss of generality that $p_{i}\left(\alpha_{4}, 1\right)=0$ for all $i \neq 4$. Do the same with $\alpha_{5}, \alpha_{6}, \ldots, \alpha_{n}$, and let

$$
\sigma:=\left(x_{1}-\alpha_{3} x_{2}\right)\left(x_{1}-\alpha_{4} x_{2}\right) \cdots\left(x_{1}-\alpha_{n} x_{2}\right) .
$$

Then

$$
\Delta^{\prime}:=\frac{\left(x_{1}-\alpha_{3} x_{2}\right) p_{3}}{\sigma} x_{3}+\frac{\left(x_{1}-\alpha_{4} x_{2}\right) p_{4}}{\sigma} x_{4}+\cdots+\frac{\left(x_{1}-\alpha_{n} x_{2}\right) p_{n}}{\sigma} x_{n}
$$


is a polynomial, and by induction on the degree, we have

$$
\Delta^{\prime}=\left|\begin{array}{cccc}
a_{3}^{(3)} & a_{4}^{(3)} & \cdots & a_{n}^{(3)} \\
a_{3}^{(4)} & a_{4}^{(4)} & \cdots & a_{n}^{(4)} \\
\vdots & \vdots & & \vdots \\
a_{3}^{(n-1)} & a_{4}^{(n-1)} & \cdots & a_{n}^{(n-1)} \\
\hline x_{3} & x_{4} & \cdots & x_{n}
\end{array}\right|
$$

with the above properties on the $a_{i}^{(j)}$. Consequently,

$$
\Delta=\left|\begin{array}{cccc}
\left(x_{1}-\alpha_{3} x_{2}\right) a_{3}^{(3)} & \left(x_{1}-\alpha_{4} x_{2}\right) a_{4}^{(3)} & \cdots & \left(x_{1}-\alpha_{n} x_{2}\right) a_{n}^{(3)} \\
\left(x_{1}-\alpha_{3} x_{2}\right) a_{3}^{(4)} & \left(x_{1}-\alpha_{4} x_{2}\right) a_{4}^{(4)} & \cdots & \left(x_{1}-\alpha_{n} x_{2}\right) a_{n}^{(4)} \\
\vdots & \vdots & & \vdots \\
\left(x_{1}-\alpha_{3} x_{2}\right) a_{3}^{(n-1)} & \left(x_{1}-\alpha_{4} x_{2}\right) a_{4}^{(n-1)} & \cdots & \left(x_{1}-\alpha_{n} x_{2}\right) a_{n}^{(n-1)} \\
\hline x_{3} & x_{4} & \cdots & x_{n}
\end{array}\right|
$$

This is essentially equivalent to Hilbert-Burch Theorem. The interested reader may wish to see [7] Theorem 20.15.

Remark 7.5. Hirokazu Nasu showed that the variety $X=(\Delta=0) \subset \mathbb{P}^{4}$ is isomorphic to the projection of the Segre variety $S \subset \mathbb{P}^{5}$ of degree three from a general point outside of $S$. Here $S \subset \mathbb{P}^{5}$ is defined as the image of the Segre embedding

$$
\mathbb{P}^{1} \times \mathbb{P}^{2} \hookrightarrow \mathbb{P}^{5}
$$

This is the locus of the maximal minors of a generic $2 \times 3$ matrix.

It is easy to see that any degree three homogeneous polynomial $F \in K\left[x_{1}, x_{2}, \Delta\right]$ in Theorem 7.3 which properly involves five variables can be transformed into the canonical form $x_{1}^{2} x_{3}+x_{1} x_{2} x_{4}+x_{2}^{2} x_{5}$ by means of a linear transformation of the variables. This form is also known as the Macaulay dual of the trivial extension of the algebra $K\left[x_{1}, x_{2}\right] /\left(x_{1}, x_{2}\right)^{3}$ by the canonical module.

\section{Proof of $\operatorname{dim} W \leq 1$ in the proof of Proposition 7.2}

We first formulate a result about homogeneous self-vanishing systems in dimension 5 in general.

Theorem 8.1. Let $\boldsymbol{h}=\left(h_{1}, h_{2}, h_{3}, h_{4}, h_{5}\right)$ be a homogeneous self-vanishing system, for which

$$
\operatorname{rank}\left(\frac{\partial h_{i}}{\partial x_{j}}\right)=\operatorname{tr} . \operatorname{deg}_{K} K(\boldsymbol{h})=\text { Krull } \operatorname{dim} K[\boldsymbol{h}] \geq 3 \text {. }
$$


Let $Z: \mathbb{P}^{4} \rightarrow \mathbb{P}^{4}$ be the rational map, defined by

$$
x=\left(x_{1}: \cdots: x_{5}\right) \mapsto\left(h_{1}: \cdots: h_{5}\right),
$$

and let $W$ be the closure of the image of $Z$.

If $\operatorname{dim} L(W)>1$, then one of the following holds.

(1) $\operatorname{dim} L(W)=3\left(\right.$ where $L(W)$ is the $K$-linear span of $W$ as a variety in $\left.\mathbb{P}^{4}\right)$.

(2) $W$ has a vertex, i.e., a point $p \in \mathbb{P}^{4}$ such that $W$ is a union of lines through $p$.

Using Theorem 8.1 , we can prove that $\operatorname{dim} W \leq 1$. Cases $(1)$ and $(2)$ are covered by Lemmas 8.2 and 8.3 respectively. Lemma 8.2 and its proof are essentially from [11, p. 567]. Lemma 8.3 is proved on [11, p. 568], and we think the proof is correct, but it could use some justification. For that reason, we formulated an alternative proof. Both Lemma 8.2 and Lemma 8.3 come from the second author's paper [4].

Lemma 8.2. Suppose that $f \in K\left[x_{1}, x_{2}, x_{3}, x_{4}, x_{5}\right]$ is homogeneous with zero Hessian, and let $\boldsymbol{h}$ be a reduced self-vanishing system arising from $f$. Then $\boldsymbol{h}$ is not as in (1) of Theorem 8.1.

Proof. Suppose that $\boldsymbol{h}$ is as in (1) of Theorem 8.1. Then $\operatorname{dim} L(W)=3$, so we may assume that the last coordinate of every point of $W$ is zero. Develop $f$ into powers of $x_{5}$, and write

$$
f=x_{5}^{\mu} A+x_{5}^{\mu+1} B,
$$

where $A \in K\left[x_{1}, x_{2}, x_{3}, x_{4}\right]$ and $B \in K\left[x_{1}, x_{2}, x_{3}, x_{4}, x_{5}\right]$. Take $g$ as in Definition 4.4. Then

$$
g\left(\frac{\partial f}{\partial x_{1}}, \frac{\partial f}{\partial x_{2}}, \frac{\partial f}{\partial x_{3}}, \frac{\partial f}{\partial x_{4}}, \frac{\partial f}{\partial x_{5}}\right)=0 .
$$

Since $g$ has minimum degree as such, $\frac{\partial g}{\partial y_{5}}=0$. Hence $g \in K\left[y_{1}, y_{2}, y_{3}, y_{4}\right]$. If we look at the trailing coefficient with respect to $x_{5}$, we see that

$$
g\left(\frac{\partial A}{\partial x_{1}}, \frac{\partial A}{\partial x_{2}}, \frac{\partial A}{\partial x_{3}}, \frac{\partial A}{\partial x_{4}}\right)
$$

i.e., $A \in K\left[x_{1}, x_{2}, x_{3}, x_{4}\right]$ has Hessian determinant zero (in dimension 4). From Theorem 7.1, it follows that we may assume that $A \in K\left[x_{1}, x_{2}, x_{3}\right]$.

If $y_{4} \mid g$, then $g=y_{4}$ because $g$ has minimum degree, so $\operatorname{deg} \boldsymbol{h}=0$. Consequently, $y_{4} \nmid g$. It follows from $A \in K\left[x_{1}, x_{2}, x_{3}\right]$ and (5) that $A \in K\left[x_{1}, x_{2}, x_{3}\right]$ has Hessian determinant zero (in dimension 3). From Theorem [5.3, it follows that we may assume that $A \in K\left[x_{1}, x_{2}\right]$.

Using Theorem 3.9 (a) and Theorem 3.11, we deduce that $f(x+t \boldsymbol{h}(x))=f(x)$. As $h_{5}=0$, we have

$$
A(x+t \boldsymbol{h}(x)) x_{5}^{\mu}+B(x+t \boldsymbol{h}(x)) x_{5}^{\mu+1}=x_{5}^{\mu} A(x)+x_{5}^{\mu+1} B(x) .
$$


If we look at the leading coefficient with respect to $t$, we see that $A(\boldsymbol{h}(x))=0$. Since $A \in K\left[x_{1}, x_{2}\right]$, we see that $h_{1}$ and $h_{2}$ are algebraically dependent over $K$. Consequently, $h_{1}$ and $h_{2}$ are linearly dependent over $K$, say that $h_{1}=0$. Then $h_{1}=h_{5}=0$, so $\operatorname{dim} L(W) \leq 2$. Contradiction.

Lemma 8.3. Suppose that $f \in K[x]=K\left[x_{1}, x_{2}, \ldots, x_{n}\right]$ is homogeneous with zero Hessian, and let $\boldsymbol{h}$ be a reduced self-vanishing system arising from $f$. Suppose that $p$ is a vertex of $W$. Then $f \in \operatorname{Sol}(p ; K[x])$. In particular, a variable can be eliminated from $f$ by way of a linear transformation of the variables.

Proof. Write $p=\left(p_{1}: p_{2}: \cdots: p_{n}\right)$ and take $d:=\operatorname{deg} \boldsymbol{h}$. Take $\tilde{\boldsymbol{h}}=\boldsymbol{h}+x_{n+1}^{d} p$. Since $p$ is a vertex of $W$, we infer that the image of

$$
\tilde{Z}: \mathbb{P}^{n}(x) \rightarrow \mathbb{P}^{n-1}(y),
$$

defined by $\left(x_{1}: x_{2}: \cdots: x_{n}: x_{n+1}\right) \mapsto\left(\tilde{h}_{1}: \tilde{h}_{2}: \cdots: \tilde{h}_{n}\right)$, is equal to $W$. Hence it follows from Lemma 5.2 that

$\operatorname{rank}\left(\partial \tilde{h}_{i} / \partial x_{j}\right)=$ Krull $\operatorname{dim} K[\tilde{\boldsymbol{h}}]=\operatorname{dim} W+1=$ Krull $\operatorname{dim} K[\boldsymbol{h}]=\operatorname{rank}\left(\partial h_{i} / \partial x_{j}\right)$.

Since $\left(\partial \tilde{h}_{i} / \partial x_{j}\right)$ can be obtained from $\left(\partial h_{i} / \partial x_{j}\right)$ by adding $\partial \tilde{\boldsymbol{h}} / \partial x_{n+1}$ as a column to the right hand side, we infer from $\operatorname{rank}\left(\partial \tilde{h}_{i} / \partial x_{j}\right)=\operatorname{rank}\left(\partial h_{i} / \partial x_{j}\right)$ that $\partial \tilde{\boldsymbol{h}} / \partial x_{n+1}=d x_{n+1}^{d-1} p$ is contained in the column space of $\left(\partial h_{i} / \partial x_{j}\right)$.

Hence $p$ is dependent over $K(x)$ on $\partial \boldsymbol{h} / \partial x_{1}, \partial \boldsymbol{h} / \partial x_{2}, \ldots, \partial \boldsymbol{h} / \partial x_{n}$. From Theorem 3.9 (c), we infer that $f \in \operatorname{Sol}(p ; K[x])$.

The rest of this section will be devoted to the proof of Theorem 8.1.

Suppose that $\operatorname{dim} W>1$, and let $T$ be the fundamental locus of $Z$ in $\mathbb{P}^{4}(x)$, defined by the equations $h_{1}(x)=h_{2}(x)=\cdots=h_{5}(x)=0$. From Theorem 3.11 (c), it follows that $\boldsymbol{h}(x+t \boldsymbol{h}(x))=\boldsymbol{h}(x)$. If we look at the leading coefficient with respect to $t$, we see that

$$
\boldsymbol{h}(\boldsymbol{h}(x))=0, \quad \text { i.e., } \quad W \subseteq T .
$$

From Proposition 6.2, it follows that $\operatorname{dim} T \leq 2$. Since $\operatorname{dim} W \geq 2$, (6) tells us that

$$
\operatorname{dim} W=\operatorname{dim} T=2 .
$$

As $W$ is the closure of the image of $Z$, we see that $W$ is a component of $T$.

On [11, p. 565], the authors claim that for every $c \in T$, there exists a $p \in W$ such that the line through $c$ and $p$ is contained in $T$. But if $c=p$, then 'the line through $c$ and $p$ ' shrinks to a single point, so it must be shown that $p$ can be taken different from $c$. The following lemma can be used for that.

Lemma 8.4. Let $\boldsymbol{h}=\left(h_{1}, h_{2}, \ldots, h_{n}\right)$ be a homogeneous self-vanishing system. Let $S$ be a hyperplane in $\mathbb{P}^{n-1}$. Then every irreducible component of $Z^{-1}(S)$ contains $W$ and has dimension $n-2$. 
Proof. We can take $\boldsymbol{\alpha}=\left(\alpha_{1}, \alpha_{2}, \ldots, \alpha_{n}\right)$, such that

$$
S=\left\{\left(\sigma_{1}: \sigma_{2}: \cdots: \sigma_{n}\right) \mid \alpha_{1} \sigma_{1}+\alpha_{2} \sigma_{2}+\cdots+\alpha_{n} \sigma_{n}=0\right\}
$$

Now $Z^{-1}(S)$ is contained in

$$
\left\{\left(\tau_{1}: \tau_{2}: \cdots: \tau_{n}\right) \mid \alpha_{1} h_{1}(\boldsymbol{\tau})+\alpha_{2} h_{2}(\boldsymbol{\tau})+\cdots+\alpha_{n} h_{n}(\boldsymbol{\tau})=0\right\},
$$

where $\boldsymbol{\tau}=\left(\tau_{1}, \tau_{2}, \ldots, \tau_{n}\right)$, and any irreducible component $X$ of $Z^{-1}(S)$ is of the form

$$
\left\{\left(\tau_{1}: \tau_{2}: \cdots: \tau_{n}\right) \mid f(\boldsymbol{\tau})=0\right\}
$$

where $f$ is an irreducible factor of $\alpha_{1} h_{1}+\alpha_{2} h_{2}+\cdots+\alpha_{n} h_{n}$. So $\operatorname{dim} X=n-2$.

It suffices to show that $f(p)=0$ for every $p$ in the image of $Z$ (i.e., skip the points of $W$ which were added by taking closure). So let $p$ be an image point of $W$. From Lemma 5.2, it follows that

$$
\operatorname{rank}\left(\frac{\partial h_{i}}{\partial x_{j}}\right)=\text { Krull } \operatorname{dim} K[\boldsymbol{h}] \leq n-1
$$

On account of the fiber dimension theorem, the closure of $Z^{-1}(p)$ has dimension at least 1. As $\operatorname{dim} X=n-2$, the intersection of $X$ and the closure of $Z^{-1}(p)$ is nonempty, say that $\theta$ is contained in this intersection.

If $\theta \in Z^{-1}(p)$, then $p=\boldsymbol{h}(\theta)$, and from Theorem 3.11 we infer that $\boldsymbol{h}(\boldsymbol{\theta}+t \boldsymbol{p})=\boldsymbol{h}(\boldsymbol{\theta})$, where $\boldsymbol{\theta}$ and $\boldsymbol{p}$ are vectors over $K$ which correspond to $\theta$ and $p$ respectively. The last equality holds in general as well because $\theta$ is contained in the closure of $Z^{-1}(p)$. Hence

$$
\operatorname{deg}_{t} f(\boldsymbol{\theta}+t \boldsymbol{p}) \leq \operatorname{deg}_{t}\left(\alpha_{1} h_{1}(\boldsymbol{\theta}+t \boldsymbol{p})+\alpha_{2} h_{2}(\boldsymbol{\theta}+t \boldsymbol{p})+\cdots+\alpha_{n} h_{n}(\boldsymbol{\theta}+t \boldsymbol{p})\right)=0 .
$$

So $f(\boldsymbol{\theta}+t \boldsymbol{p})=f(\boldsymbol{\theta})$. From $\theta \in X$, it follows that $f(\boldsymbol{\theta}+t \boldsymbol{p})=f(\boldsymbol{\theta})=0$. If we look at the leading coefficient with respect to $t$, we see that $f(\boldsymbol{p})=0$, which completes the proof.

Corollary 8.5. Let $n \geq 3$ and $\boldsymbol{h}=\left(h_{1}, h_{2}, \ldots, h_{n}\right)$ be a homogeneous self-vanishing system. Let $S$ be a hyperplane in $\mathbb{P}^{n-1}$, and $Y$ be a component of $S \cap W$. Assume that $Y$ contains an image point of $Z$. Then for every $c \in W$, there exists a $p \in Y$ such that the line through $c$ and $p$ is contained in $T$.

Proof. Let $X$ be any component of the closure of $Z^{-1}(Y)$. For every $c \in X$ for which $\boldsymbol{h}(c) \neq 0$, there exists a $p \in Y$ such that $\boldsymbol{h}(\boldsymbol{c}+t \boldsymbol{p})=\boldsymbol{h}(\boldsymbol{c})$, where $\boldsymbol{c}$ and $\boldsymbol{p}$ are vectors over $K$ which correspond to $c$ and $p$ respectively. To see this just set $p=\boldsymbol{h}(c)$ and use Theorem 3.11.

Let

$$
U=\{(c, p) \in X \times Y \mid \boldsymbol{h}(\boldsymbol{c}+t \boldsymbol{p})=\boldsymbol{h}(\boldsymbol{c})\} .
$$

Since $Y$ is a complete variety, the projection of $U$ on $X$ is a closed morphism. Hence

$$
\tilde{X}=\{c \in X \mid \text { there is a } p \in Y \text { such that } \boldsymbol{h}(\boldsymbol{c}+t \boldsymbol{p})=\boldsymbol{h}(\boldsymbol{c})\}
$$


is closed. The $c \in X$ for which $\boldsymbol{h}(c) \neq 0$ form a dense subset of $X$ and are contained in $\tilde{X}$, so $\tilde{X}=X$.

From lemma 8.4, it follows that $X$ contains $W$, so $W \subseteq \tilde{X}$. So for every $c \in W$, there exists a $p \in Y$ such that $\boldsymbol{h}(\boldsymbol{c}+t \boldsymbol{p})=\boldsymbol{h}(\boldsymbol{c})=0$, i.e., the line through $c$ and $p$ is contained in $T$.

So let us take $c \in W$. It is possible to take $S$ such that $c \notin S$ and $S$ contains an image point of $Z$. From corollary 8.5, it follows that there exist a $c^{\prime} \in S$ such that the line through $c$ and $c^{\prime}$ is contained in $T$. As $c \notin S$ and $c^{\prime} \in S$, we see that 'the line through $c$ and $c^{\prime \prime}$ does not shrink to a single point.

Since $W$ is a component of $T$, the interior $W^{\circ}$ of $W$ as a subspace of $T$ is nonempty. Now take $p \in W^{\circ}$ in the image of $Z$. There exists a $p^{\prime} \in W$ such that $p^{\prime} \neq p$ and such that the line $L_{p}$ through $p$ and $p^{\prime}$ is contained in $T$. But since $W$ is the only component of $T$ which contains $p$, it follows that $L_{p} \subseteq W$.

Taking $Y=L_{p}$ in corollary 8.5, we deduce that for every $q \in W$, there exists a $q^{\prime} \in L_{p}$ such that the line $L_{q}$ through $q$ and $q^{\prime}$ is contained in $T$. This is also claimed on [11, p. 565]. If we take $q \in W^{\circ}$, then $L_{q}$ is even contained in $W$.

Now let us fix $L_{p}$, and range $q$ over $W^{\circ}$. There are infinitely many points $q \in W^{\circ}$, but this does not mean automatically that there are infinitely many lines $L_{q}$. This is because for a line $L_{q}$, there may be infinitely many candidates for the point $q$.

However, since $\operatorname{dim} W=2$, there are infinitely many lines $L_{q}$ indeed, just as claimed on [11, p. 565]. On [11, pp. 565, 566], the following two cases (a) and (b) are distinguished:

(a) The set of points $q^{\prime} \in L_{p}$ (which we get by ranging $q$ over $W^{\circ}$ ) is infinite.

(b) There exists a fixed point $q^{\prime} \in L_{p}$, which is contained in infinitely many lines $L_{q}$.

We will treat these cases in essentially the same way as on [11, pp. 566, 568].

Assume first that case (b) above applies. Then one can show that the closure of the union of lines $L_{q}$ through $q^{\prime}$ has dimension 2. As $W$ is irreducible of dimension $2, W$ is just the closure of this union, so $W$ is a union of lines through $q^{\prime}$. Thus case (b) corresponds to case (2) of Theorem [8.1, as claimed on [11, p. 568].

Assume next that case (a) above applies. Take $r \in W^{\circ}$. Then there exists an $r^{\prime} \in L_{p}$ such that the line $L_{r}$ through $r$ and $r^{\prime}$ is contained in $W$.

Now let us fix $L_{q}$ as well, by choosing $q$ in $W^{\circ}$ in the image of $Z$ outside $L_{p}$. Range $r$ over $W^{\circ}$. On [11, p. 566], two subcases of case (a) are distinguished, which are essentially as follows:

(a1) There does not exist a line $L_{r}$ as above, such that $L_{r} \cap L_{q}=\varnothing$.

(a2) There does exist a line $L_{r}$ as above, such that $L_{r} \cap L_{q}=\varnothing$.

Both cases are treated essentially as follows on [11, p. 566].

Assume first that case (a1) above applies. Then one can show that the linear span of $p, p^{\prime}$ and $q$ contains infinitely many lines $L_{r}$, and that the closure of these lines has 
dimension 2. As $W$ is irreducible of dimension $2, W$ is just the closure of these lines, which corresponds to the linear span of $p, p^{\prime}$ and $q$.

So $\operatorname{dim} L(W) \leq 2$. Hence $\operatorname{dim} L(W)=\operatorname{dim} W$, so $L(W)=W$ and every point of $W$ is a vertex of $W$. So $W$ is as in (2) of Theorem 8.1 (and case (b) above applies as well).

Assume next that case (a2) above applies. Take $r \in W^{\circ}$ and $r^{\prime} \in L_{p}$, such that $L_{r} \cap L_{q}=\varnothing$. Take $s \in L_{r} \cap W^{\circ}$. Just as with $r^{\prime} \in L_{p}$, there exists an $s^{\prime} \in L_{q}$, such that the line $L_{s}$ through $s$ and $s^{\prime}$ is contained in $W$.

There are infinitely many lines $L_{s}$, if we range $s$ over $L_{r} \cap W^{\circ}$. Let $U$ be the closure of the lines $L_{s}$. Only finitely many lines can be a component of $U$, so there is a line which is not. Being irreducible, the line is fully contained in a component of $U$, and this component has larger dimension than the line. So $U$ has dimension at least 2. Consequently, the intersection of $W$ and the linear span of $q, q^{\prime}, r$ and $r^{\prime}$ has dimension at least 2. As $W$ is irreducible of dimension 2, $W$ is just this intersection. So $W$ is as in (2) of Theorem 8.1 ,

Remark 8.6. Self-vanishing systems corresponding to cases (a1), (a2), (b) indeed exist:

(a1) $H=\left(x_{4}^{2}, x_{4} x_{5}, x_{1} x_{5}-x_{2} x_{4}, 0,0\right)$,

(a2) $H=\left(x_{5}^{2}\left(a x_{1}-x_{5}^{2} x_{2}\right), a\left(a x_{1}-x_{5}^{2} x_{2}\right), x_{5}^{2}\left(a x_{3}-x_{5}^{2} x_{4}\right), a\left(a x_{3}-x_{5}^{2} x_{4}\right), 0\right)$ with $a=$ $x_{1} x_{4}-x_{2} x_{3}$

(b) $H=\left(x_{5}^{5}, b x_{5}^{3}, b^{2} x_{5},-b^{2} x_{1}+2 b x_{2} x_{5}^{2}-x_{3} x_{5}^{4}, 0\right)$ with $b=x_{1} x_{3}-x_{2}^{2}+x_{4} x_{5}$.

All these examples are counterexamples to the original version of Lemma 5.2. They were taken from the introduction of the second author's paper [4].

Remark 8.7. Gordan and Noether only prove that $\operatorname{dim} L(W) \leq 3$ in Theorem 8.1 (1). This makes that the conclusion that $\operatorname{dim} L(W) \leq 2$ at the end of of the proof of Lemma 8.2 is not sufficient to prove Lemma 8.2. Gordan and Noether advance as follows.

On account of $\operatorname{dim} L(W) \leq 2$, we may assume that $h_{1}=h_{2}=0$. If $f \in K\left[x_{1}, x_{2}\right]$, then $h_{i} \in K\left[x_{1}, x_{2}\right]$ for all $i$ as well, so $\operatorname{dim} W=$ Krull $\operatorname{dim} K[\boldsymbol{h}]-1=$ tr. $\operatorname{deg}_{K} K(\boldsymbol{h})-1 \leq 1$. If $f \notin K\left[x_{1}, x_{2}\right]$, then it follows from Theorem 3.9 (c) that the last three rows of the Jacobian matrix $\left(\partial h_{i} / \partial x_{j}\right)$ of $\boldsymbol{h}$ are dependent. The first two rows are zero, so $\operatorname{rank}\left(\partial h_{i} / \partial x_{j}\right) \leq 2$ and $\operatorname{dim} W=$ Krull $\operatorname{dim} K[\boldsymbol{h}]-1=\operatorname{rank}\left(\partial h_{i} / \partial x_{j}\right)-1 \leq 1$.

Remark 8.8. Theorem 8.1] is proved in [4] by the second author as well, but in a different way, because the proof by Gordan and Noether was not fully understood. The second author only proved that $\operatorname{dim} L(W) \leq 2$ in [4], because that was the missing link in [2] to obtain Theorem 7.3 .

The focus in on $\operatorname{dim} L(W)$ rather than $\operatorname{dim} W$ in other papers of the second author as well. In [3], only $\operatorname{dim} L(W) \leq 1$ is used in the classification of all homogeneous polynomials with zero Hessian in dimension 4. In [5], all homogeneous polynomials with zero Hessian in dimension 6 are classified, under the assumption that $\operatorname{dim} L(W) \leq 3$. Non-homogeneous polynomials are classified under similar assumptions in [2] and [5]. 


\section{Section 6 of Gordan and Noether [11]}

We start with formulating a result which, as opposed to Theorem 3.9, applies to $\boldsymbol{h}$ which are not constructed as in Proposition [3.8. The result is inspired by Lemma 3.1 of [13].

Proposition 9.1. Let $\boldsymbol{h}=\left(h_{1}, h_{2}, \ldots, h_{n}\right)$ be a system of forms of the same degree, and let $f \in K[x]$ be a homogeneous polynomial. Put $f_{j}=\frac{\partial f}{\partial x_{j}}$ for $j=1,2, \ldots, n$, and let $\boldsymbol{f}=\left(f_{1}, \ldots, f_{n}\right)$ Then the following statements are equivalent:

(a) $\partial \boldsymbol{h} / \partial x_{j}$ is a syzygy of $\boldsymbol{f}$ for $j=1,2, \ldots, n$.

(b) $\boldsymbol{h}$ is a syzygy of $\partial \boldsymbol{f} / \partial x_{j}$ for $j=1,2, \ldots, n$.

Furthermore, both (a) and (b) imply that $\boldsymbol{h}$ is a syzygy of $\boldsymbol{f}$.

Proof. Using

$$
\frac{\partial \boldsymbol{h}}{\partial x_{1}} x_{1}+\frac{\partial \boldsymbol{h}}{\partial x_{2}} x_{2}+\cdots+\frac{\partial \boldsymbol{h}}{\partial x_{n}} x_{n}=(\operatorname{deg} \boldsymbol{h}) \boldsymbol{h}
$$

we can obtain the last claim from (a). Similarly, the last claim can be obtained from (b). Having these results, the equivalence of (a) and (b) follows from the formula

$$
\frac{\partial}{\partial x_{j}}(\boldsymbol{h} \cdot \boldsymbol{f})=\frac{\partial \boldsymbol{h}}{\partial x_{j}} \cdot \boldsymbol{f}+\boldsymbol{h} \cdot \frac{\partial \boldsymbol{f}}{\partial x_{j}}=0 .
$$

for $j=1,2, \ldots, n$. This formula appears in the proof of Theorem 3.9 .

Let $f \in K[x]$ be a homogeneous polynomial, and put $f_{j}=\frac{\partial f}{\partial x_{j}}$. Assume that $f_{1}, f_{2}, \ldots, f_{n}$ are algebraically dependent over $K$. Then by Proposition 4.2, there exists a system $\boldsymbol{h}=\left(h_{1}, h_{2}, \ldots, h_{n}\right)$ of forms of the same degree, such that Proposition 9.1 (b) is satisfied.

Assume that

$$
h_{j}(x) \in \operatorname{Sol}(i(L(W)) ; R) \text { for all } j .
$$

Then by a linear change of variables, $\boldsymbol{h}$ coincides with a self-vanishing system described in Example 3.6. We assume this from now on, so

- $h_{1}(x)=\cdots=h_{r}(x)=0$, for some $1 \leq r<n$, and

- $h_{j}(x)$ is a function only in $x_{1}, \ldots, x_{r}$ for $j>r$.

In $\S 6$ of [11] and $\S 3$ of [13], all $f$ which satisfy Proposition 9.1 (b) are classified for this particular $\boldsymbol{h}$.

Although $\boldsymbol{h}$ is a self-vanishing system which has many properties of reduced selfvanishing systems arising from $f$, especially if $\operatorname{GCD}\left(h_{1}, h_{2}, \ldots, h_{n}\right)=1$, we will show in Example 9.5 below that it is possible for $\boldsymbol{h}$ to satisfy $\operatorname{GCD}\left(h_{1}, h_{2}, \ldots, h_{n}\right)=1$ and not to be of this form for some $f$ which satisfies Proposition 9.1 (b).

But if $\operatorname{GCD}\left(h_{1}, h_{2}, \ldots, h_{n}\right)=1$ and $\mathcal{I}(f)$ is a principal ideal, then $\boldsymbol{h}$ is indeed a reduced self-vanishing systems of $f$. This is because there is only one $\boldsymbol{h}$ with $\operatorname{GCD}\left(h_{1}, h_{2}, \ldots, h_{n}\right)=$ 1 which satisfy Proposition 9.1 (a). 
Theorem 9.2. Suppose that the Jacobian $M:=\left(\partial h_{i} / \partial x_{j}\right)$ of $\boldsymbol{h}=\left(h_{1}, h_{2}, \ldots, h_{n}\right)$ has rank $k$. Then we can choose $k$ columns of $M$ which generate its columns space, say with indices $i_{1}, i_{2}, \ldots, i_{k}$. Then $i_{1}, i_{2}, \ldots, i_{k} \in\{1,2, \ldots, r\}$.

Suppose that $f \in K\left(x_{1}, x_{2}, \ldots, x_{r}\right)\left[x_{r+1}, x_{r+2}, \ldots, x_{n}\right]$. Then $f$ is a polynomial over $K\left(x_{1}, x_{2}, \ldots, x_{r}\right)$ in the $(k+1) \times(k+1)$ minors of

$$
\left(\begin{array}{cccc}
\frac{\partial h_{r+1}}{\partial x_{i_{1}}} & \frac{\partial h_{r+2}}{\partial x_{i_{1}}} & \cdots & \frac{\partial h_{n}}{\partial x_{i_{1}}} \\
\frac{\partial h_{r+1}}{\partial x_{i_{2}}} & \frac{\partial h_{r+2}}{\partial x_{i_{2}}} & \cdots & \frac{\partial h_{n}}{\partial x_{i_{2}}} \\
\vdots & \vdots & & \vdots \\
\frac{\partial h_{r+1}}{\partial x_{i_{k}}} & \frac{\partial h_{r+2}}{\partial x_{i_{k}}} & \cdots & \frac{\partial h_{n}}{\partial x_{i_{k}}} \\
\hline x_{r+1} & x_{x+2} & \cdots & x_{n}
\end{array}\right)
$$

if and only if $f$ satisfies Proposition 9.1 (b).

Proof. Let $\tilde{K}$ be the algebraic closure of $K\left(x_{1}, x_{2}, \ldots, x_{r}\right)$. From Theorem 6.4, it follows that $f$ is a polynomial over $\tilde{K}$ in the above-described minors, if and only if $f$ satisfies Proposition 9.1 (a).

Suppose that $f$ is a polynomial over $\tilde{K}$ in the above-described minors. Let $\mathcal{B}$ be a $K\left(x_{1}, x_{2}, \ldots, x_{r}\right)$-basis of $\tilde{K}$, such that $1 \in \mathcal{B}$. Then $\mathcal{B}$ is also a basis of $\tilde{K}\left[x_{r+1}, x_{r+2}, \ldots, x_{n}\right]$ as a free module over $K\left(x_{1}, x_{2}, \ldots, x_{r}\right)\left[x_{r+1}, x_{r+2}, \ldots, x_{n}\right]$. Taking coefficients of $1 \in \mathcal{B}$ yields $f$ as a polynomial over $K\left(x_{1}, x_{2}, \ldots, x_{r}\right)$ in the above-described minors.

Remark 9.3. The proof of Theorem 6.4 tells us that the $(k+1) \times(k+1)$-minors can be replaced by $n-r-k$ homogeneous polynomials $l_{1}, l_{2}, \ldots, l_{n-r-k}$ which are linear in $x_{r+1}, x_{r+2}, \ldots, x_{n}$.

In $\S 2.3$ of [6], a similar construction is given as above, but some conditions on that construction makes it incomplete for classification. This is however not a real problem, because there is no classification result in [6]. There is just a definition of so-called GNpolynomials of type $(r, s, \mu, n)$.

Let us call a polynomial $f$ as in Theorem 9.2 a GN-polynomial of type $(n-1, n-1-$ $r, k-1)$. Then GN-polynomials of type $\left(n-1, n-1-r, k-1, n^{\prime}\right)$, as defined in [6], are GN-polynomials of type $(n-1, n-1-r, k-1)$ with additional conditions.

Remark 9.4. Since tr. $\operatorname{deg}_{K} K\left(h_{r+1}, h_{r+2}, \ldots, h_{r}\right)=k$, there exists a transcendence basis $A_{1}, A_{2}, \ldots, A_{k}$ over $K$ of $K\left(h_{r+1}, h_{r+2}, \ldots, h_{r}\right)$. In $\S 6$ of [11], $\S 3$ of [13], and $\S 2.3$ of [6], derivatives are taken with respect to $A_{1}, A_{2}, \ldots, A_{k}$ instead of $x_{i_{1}}, x_{i_{2}}, \ldots, x_{i_{k}}$ in the matrix of Theorem 9.2 ,

For other choices of $A_{1}, A_{2}, \ldots, A_{k}$, there are problems with the meaning of differentiating $h_{j}$ with respect to $A_{i}$. To obtain meaning, we choose $A_{k+1}, A_{k+2}, \ldots, A_{r}$, such that $A_{1}, A_{2}, \ldots, A_{r}$ becomes a transcendence basis of $K\left(x_{1}, x_{2}, \ldots, x_{r}\right)$. Now the only condition is that the first $k$ rows of the matrix of Theorem 9.2 are independent. With this condition, it is possible to take for $A_{1}, A_{2}, \ldots, A_{r}$ a permutation of $x_{1}, x_{2}, \ldots, x_{r}$, which is exactly what we do in Theorem 9.2 . 
Example 9.5. If $\mathcal{I}(f)$ is not a principal ideal, then is possible for $\boldsymbol{h}$ to satisfy Proposition 9.1 (b) and $\operatorname{GCD}\left(h_{1}, h_{2}, \ldots, h_{n}\right)=1$ without being a reduced self-vanishing systems arising from $f$. Take for instance

$$
f=x_{1}^{2} x_{3}+x_{1} x_{2} x_{4}+x_{2}^{2} x_{5}+z_{1}^{2} z_{3}+z_{1} z_{2} z_{4}+z_{2}^{2} z_{5}
$$

where $z_{1}, z_{2}, z_{3}, z_{4}, z_{5}=x_{6}, x_{7}, x_{8}, x_{9}, x_{10}$. Then for a reduced self-vanishing system arising from $f$, the degree which respect to $x_{1}, x_{2}, x_{3}, x_{4}, x_{5}$ has the same parity as the degree with respect to $z_{1}, z_{2}, z_{3}, z_{4}, z_{5}$. This is however not the case for

$$
\boldsymbol{h}=\left(0,0,\left(x_{1} \cdot x_{2}^{2}\right),\left(x_{1} \cdot-2 x_{1} x_{2}\right),\left(x_{1} \cdot x_{1}^{2}\right), 0,0,\left(x_{2} \cdot z_{2}^{2}\right),\left(x_{2} \cdot-2 z_{1} z_{2}\right),\left(x_{2} \cdot z_{1}^{2}\right)\right) .
$$

\section{References}

[1] W. Bruns and U. Vetter, Determinantal Rings, Lecture notes in Mathematics 1327, Springer, 1988.

[2] M.C. de Bondt and A.R.P. van den Essen, Singular Hessians, J. Algebra 282 (2004), no. $1,195-204$.

[3] M.C. de Bondt, Quasi-translations and singular Hessians, Colloq. Math. 152 (2018), no. 2, 175-198.

[4] M.C. de Bondt, Homogeneous quasi-translations in dimension 5, Beitr. Algebra Geom. 59 (2018), no. 2, 295-326.

[5] M.C. de Bondt, Polynomial Hessians with small rank, arXiv:1609.03904.

[6] C. Ciliberto, F. Russo, A. Simis, Homaloidal hypersurfaces and hypersurfaces with vanishing Hessian, Adv. Math. 218 (2008), no. 6, 1759-1805.

[7] D. Eisenbud, Commutative algebra with a view toward algebraic geometry, Graduate Texts in Mathematics 150, Springer-Verlag, New York, 1995.

[8] A. Alice, F. Repetto, A geometrical approach to Gordan-Noether's and Franchetta's contributions to a question posed by Hesse Collect. Math. 60 (2009), no. 1, 27-41.

[9] O. Hesse, Über die Bedingung, unter welcher eine homogene ganze Function von $n$ unabhängigen Variabeln durch lineäre Substitutionen von $n$ andern unabhängigen Variabeln auf eine homogene Function sich zurükfüren läfst, die eine Variable weniger enthält, Journal für die reine und angewandte Mathematik, 52 (1851), 117-124.

[10] O. Hesse, Zur Theorie der Ganzen homogenen Functionen, Journal für die reine und angewandte Mathematik, 56 (1859), 263-269.

[11] P. Gordan and M. Noether, Ueber die algebraischen Formen, deren Hesse'sche Determinante identisch verschwindet, Math. Ann. 10 (1876), 547-568. 
[12] T. Harima, J. Migliore, U. Nagel and J. Watanabe, The weak and strong Lefschetz properties for Artinian K-algebras, J. Algebra 262 (2003), 99-126.

[13] C. Lossen, When does the Hessian determinant vanish identically? Bulletin of the Brazilian Mathematical Society, 35 (1) (2004), 71-82.

[14] T. Maeno and J. Watanabe, Lefschetz elements of Artinian Gorenstein algebras and Hessians of Homogeneous polynomials, Illinois J. Mathematics, 53 (2) (2009), 591603.

[15] M. Pasch, Zur Theorie der Hesseschen Determinante, Journal für die reine und angewandte Mathematik, 80 (1875), 169-176.

[16] R-M. Miró-Roig, Determinantal ideals, Progress in Mathematics 264, Birkhäuser, 2008.

[17] J. Watanabe, A remark on the Hessian of homogeneous polynomials, in The Curves Seminar at Queen's Volume XIII, Queen's Papers in Pure and Appl. Math., 119, 2000, 171-178.

[18] J. Watanabe, On the theory of Gordan-Noether on homogeneous forms with zero Hessian, Proc. School of science of Tokai Univ., 49 (2014), 1-21.

[19] H. Yamada, On a theorem of Hesse - P. Gordan and M. Noether's Theory -, Unpublished. 